\title{
EXACT SEQUENCES AND CLOSED MODEL CATEGORIES
}

\author{
M. GARCÍA PINILLOS \\ L.J. HERNÁNDEZ PARICIO AND \\ M.T. RIVAS RODRÍGUEZ
}

\begin{abstract}
For every closed model category with zero object, Quillen gave the construction of Eckman-Hilton and Puppe sequences.

In this paper, we remove the hypothesis of the existence of zero object and construct (using the category over the initial object or the category under the final object) these sequences for unpointed model categories.

We illustrate the power of this result in abstract homotopy theory given some interesting applications to group cohomology and exterior homotopy groups.
\end{abstract}

\section{INTRODUCTION}

The usual tools of Algebraic Topology have permitted to obtain many classifications and to analyse some topological properties. However, there are families of spaces whose study requires an adaptation of the standard techniques.

For a non compact space it is advisable to consider as neighbourhoods at infinity the complements of closed-compact subsets. The Proper Homotopy Theory arises when we consider spaces and maps which are continuous at infinity. In order to have a category with limits and colimits it is interesting to extend the proper category to obtain a complete and cocomplete category. The category of exterior spaces satisfies these properties, contains the proper category and has limits and colimits. The study of non compact spaces and more generally exterior spaces has interesting applications, for example, Siebenmann [30] or Brown and Tucker [6] used proper invariants of non compact spaces to obtain some properties and classifications of open manifolds. We can also use exterior spaces to find applications in the study of compact-metric spaces. A compact metric space can be embedded in the Hilbert cube, its open neighbourhoods provide the Hilbert cube with the structure of an exterior space. In this way, the homotopy invariants of exterior spaces become invariants of metric-compact spaces.

To develop the Algebraic Topology at infinity (or in the category of exterior spaces) it is useful to consider some analogues of the standard Hurewicz homotopy groups. If instead of $n$-spheres we use sequences of $n$-spheres converging to infinity, then we obtain the Brown-Grossman proper homotopy groups, see [5], [21] . On

Date: October 1, 2008.

The authors acknowledge the financial support given by the University of La Rioja and the Government of La Rioja, project FOMENTA2007/03.

2000 Mathematics Subject Classification. 55U35, 55U40, 55N25, 55Q70.

KEY WORDS AND PHRASES. Quillen model category, model category with non-zero object, fibration sequence, cofibration sequence, group cohomology, Brown-Grossman homotopy group, Steenrod homotopy group, exterior space, proper homotopy, shape theory. 
the other hand, if we move an $n$-sphere continuously towards infinity, we get infinity semitubes which represent elements of the Steenrod proper homotopy groups, see [11], [12]. The analogues of the previous groups have been considered for the category of exterior spaces, see [17], [18]. In this homotopy theory the role of a base point is played by a base ray; that is, an exterior map from the exterior space of non negative real numbers $\mathbb{R}_{+}$to an exterior space $X$.

One of the problems that arises in the study of Steenrod and Brown-Grossman groups is that the category of exterior spaces under $\mathbb{R}_{+}$does not have a zero object and this fact implies some difficulties in the study of the exactness of some sequences or in the construction of the suspension of an exterior space, which is the base for the corresponding stable theories.

On the other hand, in the theory of Postnikov invariants of non simple spaces, for a 0 -connected space $X$ it plays an important role its first Postnikov section $X \rightarrow$ $X^{[1]}$, where $X^{[1]}$ is an Eilenberg-Mac Lane space $K(G, 1)$ with $G \cong \pi_{1}(X)$. Using cohomology with coefficients in a $G$-module $H$ (a particular case is the cohomology of a group) and working in the category of spaces over $K(G, 1)$ one can define the Postnikov invariants as elements of cohomology groups with coefficients and these invariants can be used to reconstruct the space $X$. Nevertheless, we find again the same problem: the category of spaces over $K(G, 1)$ does not have a zero object.

In order to give a common solution to these questions, in this paper we have developed some techniques that can be used to construct Eckman-Hilton and Puppe sequences in model categories without zero object. Once these constructions have been developed for an arbitrary model category, we return to the categories of exterior spaces under $\mathbb{R}_{+}$and spaces over $K(G, 1)$, and apply the properties of the corresponding Eckman-Hilton and Puppe homotopy sequences.

For example, using the homotopy sequences associated to the category of spaces over $K(G, 1)$, we obtain that the cohomology of a group $G$ with coefficients in a $G$ module $H$ can be interpreted as certain homotopy groups of twisted Eilenberg-Mac Lane spaces $(\underline{K}(G, H ; 1, n))$ in the category of spaces under and over $K(G, 1)$ :

Theorem 4.5 Let $H$ be a $G$-group and $n \geq 0$. Then

(i) $H^{n}(G ; H) \cong \pi_{0}^{r f}(\underline{\bar{K}}(G, H ; 1, n))$,

(ii) for any integer $q \geq 0, H^{n}(G ; H) \cong \pi_{q}^{r f}(\underline{K}(G, H ; 1, n+q))$.

In the case of the category of exterior spaces under $\mathbb{R}_{+}$, when we apply the homotopy sequences developed for a closed moded category without zero object, we find a long exact sequence that gives a nice connection between the two main families of exterior homotopy groups: Brown-Grossmann homotopy groups, $\pi_{q}^{B}\left(X,\left.\sigma\right|_{\mathbb{N}}\right)$, and Steenrod homotopy groups, $\pi_{q}^{S}(X, \sigma)$.

Theorem 5.2 Let $X$ be an exterior space and let $\sigma: \mathbb{R}_{+} \rightarrow X$ be a base ray, then there is an exact sequence

$$
\begin{gathered}
\cdots \rightarrow \pi_{q+1}^{B}\left(X,\left.\sigma\right|_{\mathbb{N}}\right) \rightarrow \pi_{q}^{S}(X, \sigma) \rightarrow \pi_{q}^{B}\left(X,\left.\sigma\right|_{\mathbb{N}}\right) \longrightarrow \pi_{q}^{B}\left(X,\left.\sigma\right|_{\mathbb{N}}\right) \rightarrow \\
\cdots \rightarrow \pi_{1}^{B}\left(X,\left.\sigma\right|_{\mathbb{N}}\right) \rightarrow \pi_{0}^{S}(X, \sigma) \rightarrow \pi_{0}^{B}\left(X,\left.\sigma\right|_{\mathbb{N}}\right) \longrightarrow \pi_{0}^{B}\left(X,\left.\sigma\right|_{\mathbb{N}}\right) .
\end{gathered}
$$

The long exact sequence given by Quigley [34] in Shape Theory and the sequence given by Porter [29] in proper homotopy theory can be obtained as particular cases of the long exact sequence above given for exterior spaces. 


\section{Preliminaries}

In this section, we fix the notation and recall some of the notions and results that will be used in this paper.

2.1. Closed model categories. Given a category $\mathbf{C}$, if there exists an initial object (final object) it will be denoted by $\emptyset(*)$. The opposite category is denoted by $\mathbf{C}^{o p}$. If $A$ be an object in $\mathbf{C}$, the categories under $A$ and over $A$ will be denoted by $\mathbf{C}^{A}, \mathbf{C}_{A}$, respectively. In this paper, objects and morphisms in $\mathbf{C}_{A}$ will be underlined, $\underline{f}: \underline{X} \rightarrow \underline{Y}$, and for the category under $A$ the overlined notation $\bar{f}: \bar{X} \rightarrow \bar{Y}$ will be used. A category $\mathbf{C}$ is said to be pointed if there exist initial and final objects and they are isomorphic. This object is usually denoted by $*$ and it is called the zero object.

Remark that for every category $\mathbf{C}$ one has the following properties:

(i) $\mathbf{C}^{A}$ always has initial object $\bar{A}=\mathrm{id}_{A}: A \rightarrow A$,

(ii) if $A$ is the initial object of $\mathbf{C}$, then $\mathbf{C}^{A} \cong \mathbf{C}$,

(iii) if $A$ is the final object of $\mathbf{C}$, then $\mathbf{C}^{A}$ is a pointed category, where $\bar{A}: A \rightarrow$ $A$ is the zero object.

and we also have the corresponding dual properties.

If $A$ is any given object in a category $\mathbf{C}$,

$$
\left(\mathbf{C}^{A}\right)_{\bar{A}}=\left(\mathbf{C}_{A}\right)^{\underline{A}}
$$

is a pointed category that it will also be denoted by $\mathbf{C}_{A}^{A}$. An object in this category $\underline{X}=A \stackrel{i_{X}}{\longrightarrow} X \stackrel{r_{X}}{\longrightarrow} A$ is determined by morphisms $i_{X}$ and $r_{X}$ in $\mathbf{C}$ such that $r_{X} i_{X}=\operatorname{id}_{A}$, a morphism $\underline{\bar{f}}: \underline{\bar{X}} \rightarrow \underline{\bar{Y}}$ is given by a morphism $f: X \rightarrow Y$ in $\mathbf{C}$ such that $f i_{X}=i_{Y}$ and $r_{Y} f=r_{X}$. The zero object of $\mathbf{C}_{A}^{A}$ is $\underline{\bar{A}}=A \stackrel{\operatorname{id}_{A}}{\longrightarrow} A \stackrel{\operatorname{id}_{A}}{\longrightarrow} A$.

For a given object $A$ in a category $\mathbf{C}$, we can consider the forgetful functors $U: \mathbf{C}^{A} \rightarrow \mathbf{C}$ and $V: \mathbf{C}_{A} \rightarrow \mathbf{C}$, given by $U(\bar{X})=X, U(\bar{f})=f$ and $V(\underline{X})=X$, $V(f)=f$.

If $\mathbf{C}$ has finite coproducts, then $U$ has a left adjoint $A \square(\cdot): \mathbf{C} \rightarrow \mathbf{C}^{A}$ given as follows. If $X$ is an object in $\mathbf{C}, A \bar{\square} X$ is the canonical morphism $A \square X: A \rightarrow A \sqcup X$.

Dually, if $\mathbf{C}$ has finite products, then $V$ has a right adjoint $A \underline{\times}(\cdot): \mathbf{C} \rightarrow \mathbf{C}_{A}$, which assigns to an object $X$ in $\mathbf{C}$, the object $A \times X$ which is the projection $A \times X \rightarrow A$.

The notion of closed model category, introduced by Quillen [32], is the following:

Definition 2.1. A closed model category is a category $\mathbf{C}$ endowed with three distinguished classes of morphisms called cofibrations, fibrations and weak equivalences, satisfying the following axioms CM1-CM5 below:

CM1: $\mathbf{C}$ is closed under finite limits and colimits.

CM2: If $f$ and $g$ are morphisms such that the composition $f g$ is defined then if two of them $f, g$ and $f g$ are weak equivalences, so is the third.

We say a morphism $f$ in $\mathbf{C}$ is a retract of $g$ if there are morphisms $\varphi: f \rightarrow g$ and $\psi: g \rightarrow f$ in the category of maps in $\mathbf{C}$, such that $\psi \varphi=i d$.

CM3: If $f$ is a retract of $g$, and $g$ is a cofibration, fibration or weak equivalence, then so is $f$.

A morphism which is both fibration (resp. cofibration) and weak equivalence is said to be a trivial fibration (resp. trivial cofibration). 
CM4: Given a commutative diagram of solid arrows:

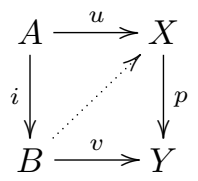

the dotted arrow exists and the triangles commute, in either of the following situations:

(i) $i$ is a cofibration and $p$ is a trivial fibration,

(ii) $i$ is a trivial cofibration and $p$ is a fibration.

CM5: Any morphism $f$ may be factored in two ways:

(i) $f=p i$, where $i$ is a cofibration and $p$ is a trivial fibration,

(ii) $f=q j$, where $j$ is a trivial cofibration and $q$ is a fibration.

If the dotted arrow exists in any diagram of the previous form $(D)$, then we say that $i: A \rightarrow B$ has the left lifting property (LLP) with respect to $p: X \rightarrow Y$, and $p$ has the right lifting property (RLP) with respect to $i$.

An object $X$ of $\mathbf{C}$ is said to be cofibrant if the unique morphism $\emptyset \rightarrow X$ is a cofibration; dually $X$ is called fibrant if $X \rightarrow *$ is a fibration. We denote by $\mathbf{C}_{c}$, $\mathbf{C}_{f}$ and $\mathbf{C}_{c f}$ the full subcategories of $\mathbf{C}$ determined by the cofibrant objects, the fibrant objects and the cofibrant and fibrant objects, respectively.

Given a closed model category $\mathbf{C}$, the category of fractions obtained by formal inversion of the weak equivalences is denoted by $\mathrm{Ho}(\mathbf{C})$, see [16], [32]. Given $X, Y$ objects in $\mathbf{C}$, we denote

$$
[X, Y]=\operatorname{Hom}_{\mathrm{Ho}(\mathbf{C})}(X, Y) .
$$

If $X$ is a cofibrant object and $Y$ is a fibrant object, then the relation of right homotopy is equal to the relation left homotopy, $\sim$, and the set of morphisms divided by the homotopy relation will be denoted by

$$
\pi[X, Y]=\operatorname{Hom}_{\mathbf{C}}(X, Y) / \sim .
$$

Moreover, in this case $[X, Y]=\pi[X, Y]$.

It is well known, see [32], the following result:

Proposition 2.1. If $\mathbf{C}$ has the structure of a closed model category, then the opposite category $\mathbf{C}^{o p}$ inherits a closed model category structure taking as cofibrations, fibrations and weak equivalences the opposites of the classes of fibrations, cofibrations and weak equivalences, respectively.

Definition 2.2. Let $\mathbf{C}$ be a closed model category and let $A$ be an object in $\mathbf{C}$. A morphism $\bar{f}$ in $\mathbf{C}^{A}$ is said to be a cofibration, a fibration or a weak equivalence if and only if $U(\bar{f})$ is a cofibration, a fibration or a weak equivalence in $\mathbf{C}$. In a dual way, a morphism $f$ in $\mathbf{C}_{A}$ is said to be a cofibration, a fibration or a weak equivalence if and only if $V(\underline{f})$ is a cofibration, a fibration or a weak equivalence in C.

We refer the reader to [32] for a proof of the following:

Proposition 2.2. Let $\mathbf{C}$ be a closed model category and let $A$ be an object in $\mathbf{C}$. Then, the categories $\mathbf{C}^{A}$ and $\mathbf{C}_{A}$, with the classes of morphisms given above, has the structure of a closed model category. 
We denote by $\operatorname{Ho}\left(\mathbf{C}^{A}\right)$ and $\operatorname{Ho}\left(\mathbf{C}_{A}\right)$, the respective localizated categories. Given $\bar{X}: A \rightarrow X$ and $\bar{Y}: A \rightarrow Y$, we will use the following notation

$$
[\bar{X}, \bar{Y}]^{A}=\operatorname{Hom}_{\mathrm{Ho}\left(\mathbf{C}^{A}\right)}(\bar{X}, \bar{Y}) .
$$

If $\bar{X}$ is cofibrant and $\bar{Y}$ is fibrant in $\mathbf{C}^{A}$; that is $\bar{X}: A \rightarrow X$ is a cofibration and $Y \rightarrow *$ is a fibration in $\mathbf{C}$, then the relation of right homotopy is equal to the relation left homotopy, $\sim$, and the set of morphisms divided by the homotopy relation will be denote by

$$
\pi[\bar{X}, \bar{Y}]^{A}=\operatorname{Hom}_{\mathbf{C}^{A}}(\bar{X}, \bar{Y}) / \sim .
$$

In this case we also have $[\bar{X}, \bar{Y}]^{A}=\pi[\bar{X}, \bar{Y}]^{A}$.

In a dual way, given $\underline{X}: X \rightarrow A$ and $\underline{Y}: Y \rightarrow A$, we will use the following notation

$$
[\underline{X}, \underline{Y}]_{A}=\operatorname{Hom}_{\mathrm{Ho}\left(\mathbf{C}_{A}\right)}(\underline{X}, \underline{Y}) .
$$

If $\underline{X}$ is cofibrant and $\underline{Y}$ is fibrant in $\mathbf{C}_{A}$; that is $\emptyset \rightarrow X$ is a cofibration and $\underline{Y}: Y \rightarrow A$ is a fibration in $\mathbf{C}$, then the relation of right homotopy is equal to the relation left homotopy, $\sim$, and the set of morphisms divided by the homotopy relation will be denote by

$$
\pi[\underline{X}, \underline{Y}]_{A}=\operatorname{Hom}_{\mathbf{C}_{A}}(\underline{X}, \underline{Y}) / \sim .
$$

In this case we also have $[\underline{X}, \underline{Y}]_{A}=\pi[\underline{X}, \underline{Y}]_{A}$.

In this paper we consider the following closed model category structure in Top: Given a map $f: X \longrightarrow Y$ in Top, $f$ is said to be a fibration if it is a fibre map in the sense of Serre; $f$ is a weak equivalence if $f$ induces isomorphism $\pi_{q}(f)$ for $q \geq 0$ and for any choice of base point and $f$ is a cofibration if it has the LLP with respect to all trivial fibrations. For this structure we refer the reader to Quillen [32]. Then, Top* inherits the following closed model category structure: A pointed map $f:(X, *) \rightarrow(Y, *)$ is said to be a fibration (resp. weak equivalence, cofibration) if in the non pointed setting the map $f: X \rightarrow Y$ is a fibration (resp. weak equivalence, cofibration). We recall that both categories of spaces and pointed spaces have compatible simplicial structures, see [32], [20] . Ho(Top), Ho(Top*) will denote the corresponding localized categories obtained by formal inversion of weak equivalences defined above.

2.2. Fibration and cofibration sequences on a pointed closed model category. Let $\mathbf{C}$ be a closed model category. For a morphism $f: X \rightarrow Y$ in $\mathbf{C}$ the fibre $F$ of $f$ in $\mathbf{C}$ is given by the pullback

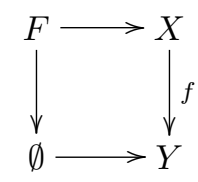

and the cofibre $C$ of $f$ in $\mathbf{C}$ is given by the pushout

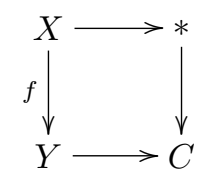


The suspension object $\Sigma X$ of $X$ is the cofibre of $X_{c} \sqcup X_{c} \rightarrow X_{c} \times I$, where $X_{c} \times I$ is a cylinder object of a cofibrant approximation $X_{c}$ of $X$. In a dual way, the loop object $\Omega X$ of $X$ is the fibre of $X_{f}^{I} \rightarrow X_{f} \times X_{f}$, where $X_{f}^{I}$ is a path object of a fibrant approximation $X_{f}$ of $X$. Thus we have induced functors $\Sigma, \Omega: \operatorname{Ho}(\mathbf{C}) \rightarrow \operatorname{Ho}(\mathbf{C})$.

Now if we suppose that $\mathbf{C}$ is a closed model category with the additional property of being pointed, then the functor $\Sigma: \mathrm{Ho}(\mathbf{C}) \rightarrow \mathrm{Ho}(\mathbf{C})$ is left adjoint to $\Omega: \operatorname{Ho}(\mathbf{C}) \rightarrow$ $\mathrm{Ho}(\mathbf{C})$. Moreover, in this case we have the following notions of fibration sequence given in $[32]$.

Definition 2.3. A diagram in $\mathrm{Ho}(\mathbf{C})$ of the form

$$
X \longrightarrow Y \longrightarrow Z \quad, X \times \Omega Z \longrightarrow X
$$

is said to be a fibration sequence in $\operatorname{Ho}(\mathbf{C})$ if there is a fibration $p: E \rightarrow B$ in $\mathbf{C}_{f}$, such that the induced diagram in $\mathrm{Ho}(\mathbf{C})$

$$
F \stackrel{i}{\longrightarrow} E \stackrel{p}{\longrightarrow} B \quad, F \times \Omega B \stackrel{m}{\longrightarrow} F
$$

is isomorphic in $\mathrm{Ho}(\mathbf{C})$ to the given diagram. Here $F$ is the fibre of $p$ and $m$ the left action of the group object $\Omega B$.

In a dual way we can consider cofibration sequences, see [32] .

For a pointed closed model category $\mathbf{C}$, Quillen [32] proved the following result:

Proposition 2.3. Let $F \stackrel{i}{\longrightarrow} E \stackrel{p}{\longrightarrow} B$ be a fibration sequence in $\mathrm{Ho}(\mathbf{C})$. Then for each object $A$ in $\mathbf{C}$ there is a sequence

$$
\begin{aligned}
& \cdots \longrightarrow\left[A, \Omega^{q+1} B\right] \stackrel{\partial_{*}}{\longrightarrow}\left[A, \Omega^{q} F\right] \stackrel{\left(\Omega^{q} i\right)_{*}}{\longrightarrow}\left[A, \Omega^{q} E\right] \stackrel{\left(\Omega^{q} p\right)_{*}}{\longrightarrow} \ldots \\
& \cdots \\
& \cdots
\end{aligned}
$$

that is exact in the following sense:

(i) $p_{*}^{-1}(0)=\operatorname{Im} i_{*}$.

(ii) $i_{*} \partial_{*}=0$ and $i_{*} \alpha_{1}=i_{*} \alpha_{2} \Longleftrightarrow \alpha_{2}=\alpha_{1} \cdot \lambda$ for some $\lambda \in[A, \Omega B]$.

(iii) $\partial_{*}(\Omega p)_{*}=0$ and $\partial_{*} \lambda_{1}=\partial_{*} \lambda_{2} \Longleftrightarrow \lambda_{2}=(\Omega p)_{*} \mu \cdot \lambda_{1}$ for some $\mu \in[A, \Omega E]$.

(iv) The sequence of group homomorphisms from $[A, \Omega E]$ to the left is exact in the usual sense.

In a dual way, one has the corresponding result for cofibration sequences.

The closed model categories has been developed very much the last decade with applications to algebraic topology and algebraic geometry. For an interesting survey you can see [9]. Some useful monographs devoted this subject are written by M. Hovey [25] , P.S. Hirschhorn [24] and W.G. Dwyer, P. S. Hirschhorn, D.M. Kan and J. Smith $[10]$. Some advances on application to algebraic topology can be seen in [7], [13], [23].

2.3. Twisted Eilenberg-Mac Lane spaces. Given a group $G$, let $K(G, 1)$ be an Eilenberg-Mac Lane space; that is, a 0-connected $\mathrm{CW}$-complex such that $\pi_{1}(K(G, 1)) \cong G$ and for $q>1, \pi_{q}(K(G, 1)) \cong 0$. Suppose that $H$ is an abelian group and $\eta: G \rightarrow \operatorname{Aut}(H)$ determines an action of $G$ on $H$, then $H$ has the structure of a $G$-module ( $\mathbf{Z} G$-module). For $n \geq 2$ denote by $K(G, H ; 1, n)$ a CW-complex such that $\pi_{1}(K(G, H ; 1, n)) \cong G, \pi_{n}(K(G, H ; 1, n)) \cong H$, for $q \notin\{1, n\} \pi_{q}(K(G, H ; 1, n))$ is trivial and the action of $\pi_{1}(K(G, H ; 1, n)) \cong G$ on $\pi_{n}(K(G, H ; 1, n)) \cong H$ is given by $\eta$. For $n=1$, we take $K(G, H ; 1,1)$ the Eilenberg-Mac Lane space $K(H \rtimes G, 1)$ associated with the semidirect product 
$H \rtimes G$ of $G$ by $H$ and for $n=0, K(G, H ; 1,0)$ is the covering space of $K(G, 1)$ induced by the representation map $\eta: G \rightarrow \operatorname{Aut}(H)$, where the covering map is denoted by $\rho_{0}: K(G, H ; 1,0) \rightarrow K(G, 1)$. We recall that for any $b \in K(G, 1)$, we have that $\rho_{0}^{-1}(b) \cong H$ (you can think on $\rho_{0}$ as a sheaf of abelian groups) and to simplify notation, we suppose that if $b_{0}$ is the base point of $K(G, 1)$, then $\rho_{0}^{-1}\left(b_{0}\right)=H$. For $h \in H$ denote by $G_{h}=\{g \in G \mid g . h=h\}$. It is well known that $\pi_{1}(K(G, H ; 1,0), h) \cong G_{h}$ and $\left.\pi_{0}(K(G, H ; 1,0)), h\right) \cong G \backslash H$, where $G \backslash H$ is the space of the orbits of the left action of $G$ on $H$.

All the spaces above (twisted Eilenberg-Mac Lane complexes) have a natural fibration $\rho_{n}: K(G, H ; 1, n) \rightarrow K(G, 1)$ and a canonical section $\sigma_{n}: K(G, 1) \rightarrow$ $K(G, H ; 1, n)$, which is a cofibration such that $\rho_{n} \sigma_{n}=\mathrm{id}_{K(G, 1)}$. We note that for each $n \geq 0, \rho_{n}: K(G, H ; 1, n) \rightarrow K(G, 1)$ is an object in the category $\mathbf{T o p}_{K(G, 1)}$ which is denoted by $\underline{K}(G, H ; 1, n)$.

Let $X$ be a 0 -connected CW-complex such that $\pi_{1}(X) \cong G$ and consider the Postnikov section $X \rightarrow X^{[1]}=K(G, 1)$. We can factor this map as

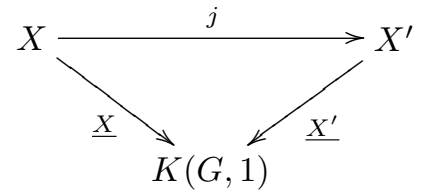

where $j$ is a trivial cofibration and $X^{\prime}$ is a fibration. Then $\underline{X}$ is isomorphic to $\underline{X^{\prime}}$ in $\operatorname{Ho}\left(\operatorname{Top}_{K(G, 1)}\right)$. Therefore if it is necessary one can suppose that the canonical map $X \rightarrow K(G, 1)$ is a fibration. For more properties about twisted Eilenberg-Mac Lane spaces we refer the reader to [1], [27].

2.4. The closed model category of exterior spaces E. One of the main applications of proper homotopy theory is the study of non compact spaces. For example, the classification of non compact surfaces given by Kérékjárto in 1923 used the notion of ideal point that can be considered as the first invariant of proper homotopy theory. Freudenthal [14] generalized this notion introducing the end point of a space and the end of a group. We can also cite the works of Siebenmann; he analyzed, in his thesis [30], the obstruction of finding a boundary to an open manifold in dimension greater than five and, in [31], he also proved important scobordism theorems. The proof of the Poincaré conjecture in dimension four was given by Freedman [15] by using s-cobordism theorems and techniques of proper homotopy theory.

We also want to mention the relationship between proper homotopy theory and shape theories, for this subject we refer the reader to [11]. For an interesting survey of the algebraic aspects of proper homotopy theory we refer the reader to [28]. We can summarize by saying that there are important applications of proper homotopy theory to the study of manifolds, ends of a group, shape and prohomotopy theories, etc.

Let $X$ and $Y$ be topological spaces. A continuous map $f: X \rightarrow Y$ is said to be proper if for every closed compact subset $K$ of $Y, f^{-1}(K)$ is a compact subset of $X$. The category of spaces and proper maps will be denoted by $\mathbf{P}$.

One of the main problems of the proper category is that there are few limits and colimits. By this reason, it is difficult to develop some homotopy constructions as homotopy fibres or loop spaces. 
In [18], [17] there is a solution for this problem introducing the notion of exterior space. The category of exterior spaces and exterior maps, $\mathbf{E}$, is complete and cocomplete and contains $\mathbf{P}$ as a full subcategory. Furthermore, $\mathbf{E}$ has a closed simplicial model category structure in the sense of Quillen [32] ; hence, it establishes a good framework for the study of proper homotopy theory.

The localized category $\mathrm{Ho}(\mathbf{E})$ has also interesting applications to shape and strong shape theory, see [17]. In shape theory, the role of Hurewicz homotopy groups is played by homotopy progroups, see [11], [26] or equivalently by BrownGrossmann homotopy groups, see [21]. In strong shape theory, one of the main strong shape invariants are the Steenrod homotopy groups, see [11].

We recall in this subsection the notion of exterior space. Roughly speaking, an exterior space is a topological space $X$ with a neighbourhood system at infinity.

Definition 2.4. An exterior space (or exterior topological space) $(X, \varepsilon \subset \tau)$ consists of a topological space $(X, \tau)$ together with a non empty collection $\varepsilon$ of open subsets satisfying,

E1: If $E_{1}, E_{2} \in \varepsilon$ then $E_{1} \cap E_{2} \in \varepsilon$,

E2: if $E \in \varepsilon, U \in \tau$ and $E \subset U$, then $U \in \varepsilon$.

An open $E$ which is in $\varepsilon$ is said to be an exterior-open subset or, briefly, an eopen subset. The family of e-open subsets $\varepsilon$ is called the externology of the exterior space. A map $f:(X, \varepsilon \subset \tau) \rightarrow\left(X^{\prime}, \varepsilon^{\prime} \subset \tau^{\prime}\right)$ is said to be exterior if it is continuous and $f^{-1}(E) \in \varepsilon$, for all $E \in \varepsilon^{\prime}$.

Given an space $(X, \tau)$, we can always consider the trivial exterior space taking $\varepsilon=\{X\}$ and the total exterior space if one takes $\varepsilon=\tau$. An important role is played by the family $\varepsilon_{c c}^{X}$ of the complements of closed-compact subsets of a topological space $X$, that will be called the cocompact externology. We denote by $\mathbb{N}$ and $\mathbb{R}_{+}$ the exterior spaces of non negative integers and non negative real numbers having the usual topology and the cocompact externology.

The full embedding $e: \mathbf{P} \hookrightarrow \mathbf{E}$ carries an space $X$ to the exterior space $X_{e}$ which is provided with the topology of $X$ and $\varepsilon_{c c}^{X}$. A proper map $f: X \rightarrow Y$ is carried to the exterior map $f_{e}: X_{e} \rightarrow Y_{e}$ given by $f_{e}=f$.

Definition 2.5. Let $X$ be an exterior space, $Y$ a topological space. Consider on $X \times Y$ the product topology and the distinguished open subsets $E$ of $X \times Y$ such that for each $y \in Y$ there exists $U_{y} \in \tau_{Y}, y \in U_{y}$ and $E_{y} \in \varepsilon_{X}$ such that $E_{y} \times U_{y} \subset E$. This exterior space will be denoted by $X \overline{\times} Y$.

This construction gives a functor $\mathbf{E} \times \mathbf{T o p} \rightarrow \mathbf{E}$, where Top denotes the category of topological spaces. When $Y$ is a compact space then $E$ is an e-open subset of $X \overline{\times} Y$ if and only if it is an open subset and there exists $G \in \varepsilon_{X}$ such that $G \times Y \subset E$. Furthermore, if $Y$ is a compact space and $\varepsilon_{X}=\varepsilon_{c c}^{X}$ then $\varepsilon_{X \overline{\times} Y}=\varepsilon_{c c}^{X \times Y}$.

Let $\mathbf{E}^{\mathbb{N}}$ be the category of exterior spaces under $\mathbb{N}$. In this context, it is usual to denote an object by a pair $(X, \rho)$, where $\rho: \mathbb{N} \rightarrow X$ is an exterior map, which is called a base sequence in $X$. A morphisms in $\mathbf{E}^{\mathbb{N}}$ given by a commutative triangle in $\mathbf{E}$

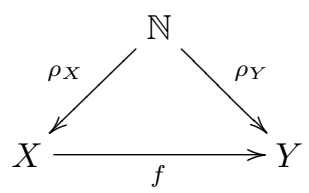


is usually denoted by $f:\left(X, \rho_{X}\right) \rightarrow\left(Y, \rho_{Y}\right)$.

Definition 2.6. Let $f, g$ be in $\operatorname{Hom}_{\mathbf{E}^{\mathbb{N}}}\left(\left(X, \rho_{X}\right),\left(Y, \rho_{Y}\right)\right)$, then we say $f$ is e-homotopic to $g$ relative to $\mathbb{N}$, written $f \simeq_{e}^{\mathbb{N}} g$, if there is an exterior map $F$ : $X \overline{\times} I \rightarrow Y$ such that $F(x, 0)=f(x), F(x, 1)=g(x)$ and $F\left(\rho_{X}(k), t\right)=\rho_{Y}(k)$, for all $x \in X, k \in \mathbb{N}$ and $t \in I$. The map $F$ is called an exterior homotopy relative to $\mathbb{N}$ from $f$ to $g$ and we sometimes write $F: f \simeq_{e}^{\mathbb{N}} g$.

In [17], it is proved that $\operatorname{Hom}_{\mathbf{E}^{\mathbb{N}}}\left(\left(\mathbb{N} \overline{\times} S^{q}, i d_{\mathbb{N}} \overline{\times} *\right),(X, \rho)\right) / \simeq_{e}^{\mathbb{N}}$ has the structure of a group for $q \geq 1$, which is abelian for $q \geq 2$; if $q=0$ we get a pointed set.

Definition 2.7. Let $(X, \rho)$ be an object of $\mathbf{E}^{\mathbb{N}}$. For $q \geq 0$, the q-th BrownGrossman exterior homotopy group of $(X, \rho)$ is given by

$$
\pi_{q}^{B}(X, \rho)=\operatorname{Hom}_{\mathbf{E}^{\mathbb{N}}}\left(\left(\mathbb{N} \overline{\times} S^{q}, i d_{\mathbb{N}} \bar{x} *\right),(X, \rho)\right) / \simeq_{e}^{\mathbb{N}} .
$$

In an analogous way, one can consider the category $\mathbf{E}^{\mathbb{R}_{+}}$of exterior spaces under $\mathbb{R}_{+}$. In this case, an object in $\mathbf{E}^{\mathbb{R}_{+}}$will be denoted by $(X, \sigma)$, where $\sigma: \mathbb{R}_{+} \rightarrow X$ is an exterior map, which is called a base ray in $X$. A morphism in $\mathbf{E}^{\mathbb{R}_{+}}$will be denoted by $f:\left(X, \sigma_{X}\right) \rightarrow\left(Y, \sigma_{Y}\right)$. Similarly, we define the exterior homotopy relation relative to $\mathbb{R}_{+}, \simeq_{e}^{\mathbb{R}_{+}}$. Then, see [18], the set of exterior homotopy classes relative to $\mathbb{R}_{+}, \operatorname{Hom}_{\mathbf{E}^{\mathbb{R}_{+}}}\left(\left(\mathbb{R}_{+} \overline{\times} S^{q}, i d_{\mathbb{R}_{+}} \overline{\times} *\right),(X, \sigma)\right) / \simeq_{e}^{\mathbb{R}_{+}}$admits the structure of a group for $q \geq 1$, which is abelian if $q \geq 2$; and for $q=0$ a pointed set is obtained.

Definition 2.8. Let $(X, \sigma)$ be an object of $\mathbf{E}^{\mathbb{R}_{+}}$. For every $q \geq 0$, the q-th Steenrod exterior homotopy group of $(X, \sigma)$ is given by

$$
\pi_{q}^{S}(X, \sigma)=\operatorname{Hom}_{\mathbf{E}^{\mathbb{R}_{+}}}\left(\left(\mathbb{R}_{+} \overline{\times} S^{q}, i d_{\mathbb{R}_{+}} \overline{\times} *\right),(X, \sigma)\right) / \simeq_{e}^{\mathbb{R}_{+}} .
$$

In order to obtain exact sequences for exterior Brown-Grossman and Steenrod homotopy groups, we will consider in the category $\mathbf{E}$ the closed model structure given by the following classes of maps.

Definition 2.9. Let $f: X \rightarrow Y$ be an exterior map.

(i) $f$ is a exterior weak equivalence, if either

(a) if $\operatorname{Hom}_{\mathbf{E}}(\mathbb{N}, X)=\emptyset$ then $\operatorname{Hom}_{\mathbf{E}}(\mathbb{N}, Y)=\emptyset$, or

(b) if $\operatorname{Hom}_{\mathbf{E}}(\mathbb{N}, X) \neq \emptyset$ then $\pi_{q}^{B}(f): \pi_{q}^{B}(X, \rho) \rightarrow \pi_{q}^{B}(Y, f \rho)$ is an isomorphism for all $\rho \in \operatorname{Hom}_{\mathbf{E}}(\mathbb{N}, X), q \geq 0$.

(ii) $f$ is an exterior fibration if it has the $R L P$ with respect to $\partial_{0}: \mathbb{N} \overline{\times} D^{q} \rightarrow$ $\mathbb{N} \overline{\times}\left(D^{q} \times I\right)$ for all $q \geq 0$, where $\partial_{0}(n, x)=(n, x, 0)$.

$A$ map which is both an exterior fibration and an exterior weak equivalence is said to be an exterior trivial fibration.

(iii) $f$ is an exterior cofibration if it has the LLP with respect to any exterior trivial fibration.

A map which is both an exterior cofibration and an exterior weak equivalence is said to be an exterior trivial cofibration.

Then, see [17], one has the following result.

Theorem 2.1. The category of exterior spaces, E, together with the classes of exterior fibrations, exterior cofibrations and exterior weak equivalences has a closed simplicial model category structure.

To see recient applications of the category of exterior spaces to proper homotopy theory we refer the reader to [19]. 


\section{LONG EXACT SEQUENCES IN AN ARBITRARY ClOSED MODEL CATEGORY}

Given a closed model category $\mathbf{C}$, then one has induced closed model structures in the category over the initial object $\emptyset$ and in the category under the final object * We use the fact that these relative and fibre categories have zero object to construct useful long exact sequences.

3.1. The categories over $A$ and under $A$. In this section we suppose that $\mathbf{C}$ is a closed model category and $A$ is an object in $\mathbf{C}$; by Proposition 2.2 one has that $\mathbf{C}_{A}$ and $\mathbf{C}^{A}$ inherit the structure of a closed model category.

Next we analyse some properties of the adjoint functores $V$ and $A \times(\cdot)$ with respect to the model structures. Recall that if $\mathbf{C}, \mathbf{C}^{\prime}$ are closed model categories, and $F: \mathbf{C} \rightarrow \mathbf{C}^{\prime}$ is a functor which preserves weak equivalences between cofibrant objects, $F$ induces a left derived functor $F^{L}: \operatorname{Ho}(\mathbf{C}) \rightarrow \operatorname{Ho}\left(\mathbf{C}^{\prime}\right)$ given by $F^{L}(X)=$ $F\left(X_{c}\right)$, where $X_{c}$ is a cofibrant approximation of $X$ (dually we have right derived functors), see [32].

Theorem 3.1. Let $\mathbf{C}$ be a closed model category and suppose that the object $A$ is fibrant. Then the functors $V: \mathbf{C}_{A} \rightarrow \mathbf{C}$ and $A \times(\cdot): \mathbf{C} \rightarrow \mathbf{C}_{A}$ induce on the localized categories the functors $V^{L}: \operatorname{Ho}\left(\mathbf{C}_{A}\right) \rightarrow \operatorname{Ho}(\overline{\mathbf{C}})$ and $(A \times(\cdot))^{R}: \operatorname{Ho}(\mathbf{C}) \rightarrow \operatorname{Ho}\left(\mathbf{C}_{A}\right)$ where $V^{L}$ is the left derived functor and $(A \times(\cdot))^{R}$ is the right derived functor. The induced functors satisfy that $V^{L}$ is left adjoint to $(A \underline{\times}(\cdot))^{R}$ (briefly they will also be denoted by $V$ and $A \underline{\times}(\cdot))$.

Proof. We will apply Theorem 3 of Section 4 of Chapter I from [32]. For this purpose it suffices to see that $V$ preserves cofibrations and carries weak equivalences between cofibrant objects in $\mathbf{C}_{A}$ to weak equivalences in $\mathbf{C}$, and that $A \underline{\times}(\cdot)$ preserves fibrations and carries weak equivalences between fibrant objects to weak equivalences in $\mathbf{C}_{A}$.

From Definition 2.2 it follows that $V$ preserves cofibrations, fibrations and weak equivalences. Since $A \underline{\times}(\cdot)$ is right adjoint to $V$, it follows that $A \underline{\times}(\cdot)$ preserves fibrations.

Suppose that $f: X \rightarrow Y$ be a weak equivalence between fibrant objects in $\mathbf{C}$, since $A$ is fibrant, we have that $\operatorname{id}_{A} \times f: A \times X \rightarrow A \times Y$ is a weak equivalence between fibrant objects in $\mathbf{C}$. Taking into account that $\operatorname{id}_{A} \times f=V(A \times f)$, we obtain that $A \times f$ is a weak equivalence in $\mathbf{C}_{A}$. To see that $\operatorname{id}{ }_{A} \times f$ is a weak equivalence you can apply Proposition 2.6 of chapter I and the dual of Lemma 1.2 of Chapter II of [2] . An alternative proof can be obtained using the fact that $\mathrm{Ho}(\mathbf{C})$ has finite products.

In the following results we use notions and notations given by Quillen in section 2 of Chapter I of [32] about left homotopies, right homotopies, cylinders, left homotopies between left homotopies, et cetera, in a closed model category $\mathbf{C}$ which needn't be pointed. In particular if $X$ is cofibrant and $Y$ is fibrant and $f, g: X \rightarrow Y$ are morphisms in $\mathbf{C}, \pi_{l}(X, Y ; f, g)$ denotes the set of homotopy classes of left homotopies from $f$ to $g$.

Proposition 3.1. Let $A$ be a fibrant object in $\mathbf{C}$. Suppose that $\underline{Z}$ in $\mathbf{C}_{A}$ satisfies that $[V \underline{Z}, A]=*$ and $\pi_{l}(V \underline{Z}, A ; \underline{Z}, \underline{Z})=*$. Then for any object $\underline{F}$ in $\mathbf{C}_{A}$, we have that $[\underline{Z}, \underline{F}]_{A} \cong[V \underline{Z}, V \underline{F}]$. 
Proof. Firstly, since each object has cofibrant and fibrant approximations, we can assume that $\underline{Z}$ is cofibrant and $\underline{F}$ is fibrant in $\mathbf{C}_{A}$. If $V \underline{Z}=Z$ and $V \underline{F}=F$, this is equivalent to say that $Z$ is cofibrant and $F: F \rightarrow A$ is a fibration in $\mathbf{C}$. Since $A$ is fibrant, so $F$ is also fibrant in $\mathbf{C}$. We recall that a map in $[\underline{Z}, \underline{F}]_{A}$ can be represented by the homotopy class $[f]_{A}$ of a map $\underline{f}: \underline{Z} \rightarrow \underline{F}$ given by the following commutative diagram

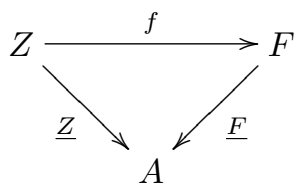

We consider the map $\varphi:[\underline{Z}, \underline{F}]_{A} \rightarrow[V \underline{Z}, V \underline{F}]$ given by $\varphi\left([f]_{A}\right)=[V \underline{f}]$. First we will see that $\varphi$ is surjective. Given $f: Z \rightarrow F$ Since $[Z, \bar{A}]=*$, there is a homotopy $G: Z \times I \rightarrow A$ such that $G \partial_{0}=\underline{F f}$ and $G \partial_{1}=\underline{Z}$, where

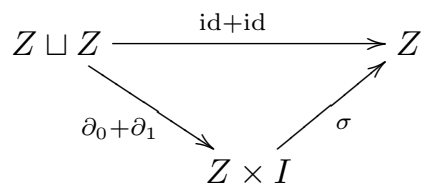

is the cylinder of $Z$.

Now we can consider the commutative diagram

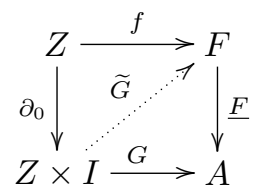

where the lift $\widetilde{G}$ exists because $F$ is fibration and $\partial_{0}$ is a trivial cofibration. Moreover, $\underline{F} \widetilde{G} \partial_{1}=G \partial_{1}=\underline{Z}$ so we have $\left[\underline{\widetilde{G}} \partial_{1}\right]_{A} \in[\underline{Z}, \underline{F}]_{A}$ verifying $\varphi\left(\left[\underline{\widetilde{G}} \partial_{1}\right]_{A}\right)=\left[\widetilde{G} \partial_{1}\right]=$ $[f]$.

Next we prove that $\varphi$ is injective: Let $[f]_{A},\left[f^{\prime}\right]_{A} \in[\underline{Z}, \underline{F}]_{A}$ such that $\varphi\left([f]_{A}\right)=$ $\varphi\left(\left[f^{\prime}\right]_{A}\right)$, that is $f \sim f^{\prime}$ in $\mathbf{C}$. Let $H: \bar{Z} \times \bar{I} \rightarrow F$ be a homotopy such that $H \partial_{0}=f, H \partial_{1}=f^{\prime}$. We note that $\underline{F} H$ and $\underline{Z} \sigma$ are left homotopies from $\underline{Z}$ to $\underline{Z}$ in $\mathbf{C}$. We can consider $Z \times I \underset{Z \sqcup Z}{\sqcup} Z \times I$ as the push-out of $\partial_{0}+\partial_{1}$ and $\partial_{0}+\partial_{1}$. Now if we factor the map $\Delta=\mathrm{id}+\mathrm{id}$ as the composition of a cofibration $j_{0}+j_{1}$ and a trivial fibration $\bar{\sigma}$ we obtain the following commutative diagram:

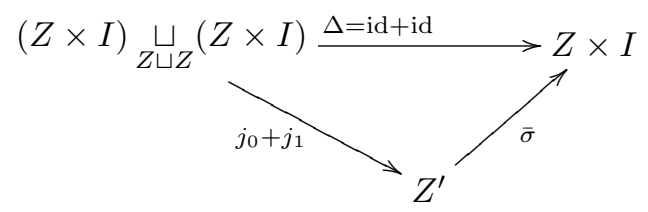


Since $\pi_{l}(V \underline{Z}, A ; \underline{Z}, \underline{Z})=*$, there is a map $L: Z^{\prime} \rightarrow A$ such that the following diagram is commutative:

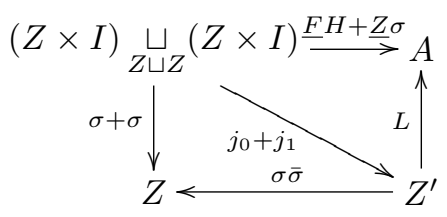

Since $j_{0}$ is a trivial cofibration and $\underline{F}$ is a fibration, there is $\tilde{L}: Z^{\prime} \rightarrow F$ such that the following diagram is commutative

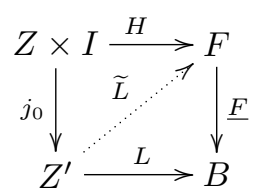

We note that $\underline{F} \tilde{L} j_{1}=\underline{Z} \sigma$, then $\tilde{L} j_{1}$ is a morphism in $\mathbf{C}_{A}$ from $\underline{Z} \sigma$ to $\underline{F}$. Since $\tilde{L} j_{1} \partial_{0}=\tilde{L} j_{0} \partial_{0}=H \partial_{0}=f, \tilde{L} j_{1} \partial_{1}=\bar{L} j_{0} \partial_{1}=H \partial_{1}=f^{\prime}$, then $\tilde{L} j_{1}$ is a homotopy from $\underline{f}$ to $f^{\prime}$ in $\mathbf{C}_{A}$.

Lemma 3.1. Let $A$ be a fibrant and cofibrant object in $\mathbf{C}$. Suppose that $\underline{Z}$ is cofibrant in $\mathbf{C}_{A}$. Then

(i) If $[V(\Sigma \underline{Z}), A]=*$, implies $\pi_{l}(V \underline{Z}, A ; \underline{Z}, \underline{Z})=*$.

(ii) In the case $A=\emptyset$, we also have that $[V(\Sigma \underline{Z}), A]=*$ if and only if $\pi_{l}(V \underline{Z}, A ; \underline{Z}, \underline{Z})=*$.

Proof. The suspension $\Sigma \underline{Z}$ of the cofibrant object $\underline{Z}: Z \rightarrow A$ in $\mathbf{C}_{A}$ is induced by the following pushout

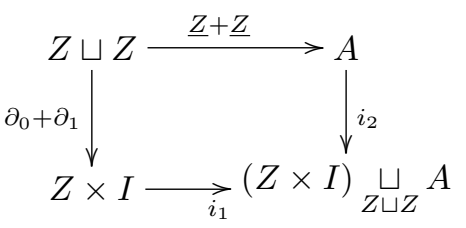

as the morphism

$$
\Sigma \underline{Z}:(Z \times I) \underset{Z \sqcup Z}{\sqcup} A \stackrel{\underline{Z} \sigma+\mathrm{id}_{A}}{\longrightarrow} A
$$

Notice that since $A$ is cofibrant, $V(\Sigma \underline{Z})$ is cofibrant in $\mathbf{C}$.

Let $h, h^{\prime}: Z \times I \rightarrow A$ be two left homotopies between $\underline{Z}$ and $\underline{Z}$. Then $h, h^{\prime}$ induce $k, k^{\prime}: V(\Sigma \underline{Z}) \rightarrow A$ given by $k=h+\mathrm{id}_{A}, k^{\prime}=h^{\prime}+\mathrm{id}_{A}$. Since $[V(\Sigma \underline{Z}), A]=*$, there is a right homotopy $M: V(\Sigma \underline{Z}) \rightarrow A^{I}$ between $k, k^{\prime}$, where

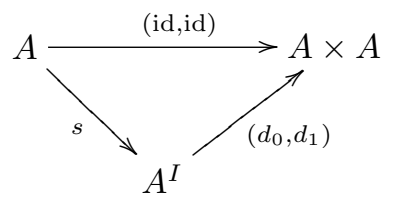

is the cocylinder of $A$. 
Consider a correspondence $H: Z \times I \rightarrow A^{I}$ from $h^{\prime}$ to a right homotopy $l^{\prime}: Z \rightarrow$ $A^{I}$ between $\underline{Z}$ and $\underline{Z}$.

Using $H$ and $M i_{1}$, one has that $h, h^{\prime}$ correspond to $s \underline{Z} . l^{\prime}, l^{\prime}$, respectively. Since $s \underline{Z} . l^{\prime}$ and $l^{\prime}$ represent the same class, it follows that $h, h^{\prime}$ represent the same element in $\pi_{l}(Z, A ; \underline{Z}, \underline{Z})$.

In order to prove (ii), in the case $A=\emptyset$, let $k, k^{\prime}: V(\Sigma \underline{Z}) \rightarrow \emptyset$, then $h, h^{\prime}: Z \times \emptyset$ given by $h=k i_{1}, h^{\prime}=k^{\prime} i_{1}$ are left homotopies from $\underline{Z}$ to $\underline{Z}$ and $k=h+\operatorname{id}_{\emptyset}$, $k^{\prime}=h^{\prime}+\mathrm{id}_{\emptyset}$ since $k i_{2}=k^{\prime} i_{2}=\mathrm{id}_{\emptyset}$.

Taking into account $\pi_{l}(Z, \emptyset ; \underline{Z}, \underline{Z}) \cong *$, there is $P: Z^{\prime} \rightarrow \emptyset$ a left homotopy from $h$ to $h^{\prime}$. Take $P: Z^{\prime} \rightarrow \emptyset$ a left homotopy from $h$ to $h$.

Since $j_{0}$ is a trivial cofibration and $\left(d_{0}, d_{1}\right)$ is a fibration, there is $N: Z^{\prime} \rightarrow \emptyset^{I}$ such that the following diagram is commutative

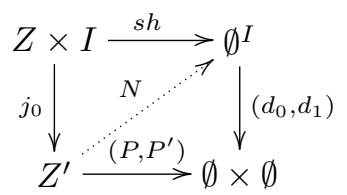

Taking $T=N j_{1}+s: V(\Sigma \underline{Z}) \rightarrow \emptyset^{I}$ we have a right homotopy from $k$ to $k^{\prime}$.

Corollary 3.1. If $\emptyset$ is a fibrant object in $\mathbf{C}$ and $\underline{Z}$ in $\mathbf{C}_{\emptyset}$ satisfies that $[V \underline{Z}, \emptyset]=$ * and $[V(\Sigma \underline{Z}), \emptyset]=*$, then for any object $\underline{F}$ in $\mathbf{C}_{\emptyset}$, we have that $[\underline{Z}, \underline{F}]_{\emptyset} \cong$ $[V \underline{Z}, V \underline{F}]$.

Dually, we analyse the corresponding properties of the adjoint functors $A \square(\cdot)$ and $U$ with respect to the model structures in $\mathbf{C}$ and $\mathbf{C}^{A}$.

Theorem 3.2. Let $\mathbf{C}$ be a closed model category and suppose that the object $A$ is cofibrant. Then the functors $A \square(\cdot): \mathbf{C} \rightarrow \mathbf{C}^{A}$ and $U: \mathbf{C}^{A} \rightarrow \mathbf{C}$ induce on the localized categories the functors $(A \square(\cdot))^{L}: \mathrm{Ho}(\mathbf{C}) \rightarrow \mathrm{Ho}\left(\mathbf{C}^{A}\right)$ and $U^{R}: \mathrm{Ho}\left(\mathbf{C}^{A}\right) \rightarrow$ $\operatorname{Ho}(\mathbf{C})$ where $(A \square(\cdot))^{L}$ is the left derived functor and $U^{R}$ is the right derived functor. The induced functors satisfy that $(A \square(\cdot))^{L}$ is left adjoint to $U^{R}$ (briefly they will also be denoted by $A \square(\cdot)$ and $U)$.

Proposition 3.2. Let $A$ be a cofibrant object in $\mathbf{C}$. Suppose that $\bar{Z}$ in $\mathbf{C}^{A}$ satisfies that $[A, U \bar{Z}]=*$ and $\pi_{l}(A, U \bar{Z} ; \bar{Z}, \bar{Z})=*$. Then for any object $\bar{F}$ in $\mathbf{C}^{A}$, we have that $[\bar{F}, \bar{Z}]^{A} \cong[U \bar{F}, U \bar{Z}]$.

Lemma 3.2. Let $A$ be a cofibrant and fibrant object in $\mathbf{C}$. Suppose that $\bar{Z}$ is fibrant in $\mathbf{C}^{A}$. Then

(i) If $[A, U(\Omega \bar{Z})]=*$, implies $\pi_{l}(A, U \bar{Z} ; \bar{Z}, \bar{Z})=*$.

(ii) In the case $A=*$, we also have that $[A, U(\Omega \bar{Z})]=*$ if and only if $\pi_{l}(A, U \bar{Z} ; \bar{Z}, \bar{Z})=*$.

Corollary 3.2. If $*$ is a cofibrant object in $\mathbf{C}$ and $\bar{Z}$ in $\mathbf{C}^{*}$ satisfies that $[*, U \bar{Z}]=*$ and $[*, U(\Omega \bar{Z})]=*$, then for any object $\bar{F}$ in $\mathbf{C}^{*}$, we have that $[\bar{F}, \bar{Z}]^{*} \cong[U \bar{F}, U \bar{Z}]$.

3.2. Exact sequences. For a map $f: X \rightarrow Y$ in a closed model category $\mathbf{C}$ we use the model structure of $\mathbf{C}_{\emptyset}$ to construct exact sequences associated with $f$. 
Given a map $p: E \rightarrow B$ in $\mathbf{C}$, the "fibre" of $p$ in $\mathbf{C}_{\emptyset}$ is the object $\underline{F}: F \rightarrow \emptyset$ given by the following pullback

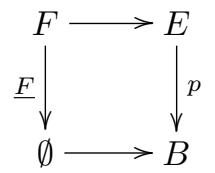

It is interesting to note that the "fibre" $\underline{F}$ of $p$ in $\mathbf{C}_{\emptyset}$ is isomorphic to the fibre of $\emptyset \times p$ in the pointed category $\mathbf{C}_{\emptyset}$. On the other hand, if $f$ is a morphism in $\mathbf{C}_{\emptyset}$, the fibre of $f$ is the "fibre" of $V \underline{f}$.

For a given map $f: X \rightarrow Y$ in $\mathbf{C}$, we can consider a commutative diagram

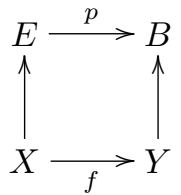

where $p$ is a fibration, $E, B$ are fibrant and the vertical maps are trivial cofibrations. Then $p$ is isomorphic to $f$ in the localized category and it is said that $p$ is an approaching fibration of the map $f$.

Definition 3.1. Let $\mathbf{C}$ be a closed model category such that $\emptyset$ is fibrant. If $p: E \rightarrow B$ is an approaching fibration of a morphism $f: X \rightarrow Y$ in $\mathbf{C}$, the "fibre" of $p$ in $\mathbf{C}_{\emptyset}$ is said to be the "homotopy fibre" of $f$ in $\mathbf{C}_{\emptyset}$ and it will be denoted by $\underline{F(f)}$.

Notice that all possible "homotopy fibres" of $f$ in $\mathbf{C}_{\emptyset}$ are weak equivalent.

Theorem 3.3. Let $\mathbf{C}$ be a closed model category such that $\emptyset$ is fibrant. Let $f: X \rightarrow Y$ a morphism in $\mathbf{C}$ and let $F(f): F(f) \rightarrow \emptyset$ be the "homotopy fibre" of $f$ in $\mathbf{C}_{\emptyset}$ and $\underline{Z}: Z \rightarrow \emptyset$ an object in $\overline{\mathbf{C}_{\emptyset} \text {. Then }}$

(i) there is a long exact sequence

$\cdots \longrightarrow\left[\underline{Z}, \Omega^{q+1}(\emptyset \times Y)\right]_{\emptyset} \rightarrow\left[\underline{Z}, \Omega^{q}(F(f))\right]_{\emptyset} \rightarrow\left[\underline{Z}, \Omega^{q}(\emptyset \times X)\right]_{\emptyset} \rightarrow \cdots$

$\cdots \rightarrow[\underline{Z}, \Omega(\emptyset \times X)]_{\emptyset} \rightarrow[\underline{Z}, \Omega(\emptyset \times Y)]_{\emptyset} \rightarrow[\underline{Z}, F(f)]_{\emptyset} \rightarrow[\underline{Z}, \emptyset \times X]_{\emptyset} \rightarrow[\underline{Z}, \emptyset \times Y]_{\emptyset}$

(ii) the sequence given at (i) is isomorphic to the exact sequence

$\cdots \longrightarrow\left[V \Sigma^{q+1} \underline{Z}, Y\right] \longrightarrow\left[\Sigma^{q} \underline{Z}, F(f)\right]_{\emptyset} \longrightarrow\left[V \Sigma^{q} \underline{Z}, X\right] \longrightarrow \cdots$

$\cdots \longrightarrow[V \Sigma \underline{Z}, X] \longrightarrow[V \Sigma \underline{Z}, Y] \longrightarrow[\underline{Z}, F(f)]_{\emptyset} \longrightarrow[Z, X] \longrightarrow[Z, Y]$

(iii) If $\underline{Z}$ satisfies that $\left[V\left(\Sigma^{k} \underline{Z}\right), \emptyset\right]=*$, for $k \geq 0$, then the sequences given at (i) and (ii) are isomorphic to the exact sequence

$$
\begin{aligned}
& \cdots \longrightarrow\left[V \Sigma^{q+1} \underline{Z}, Y\right] \longrightarrow\left[V \Sigma^{q} \underline{Z}, F(f)\right] \longrightarrow\left[V \Sigma^{q} \underline{Z}, X\right] \longrightarrow \cdots \\
& \cdots \longrightarrow[V \Sigma \underline{Z}, X] \longrightarrow[V \Sigma \underline{Z}, Y] \longrightarrow[Z, F(f)] \longrightarrow[Z, X] \longrightarrow[Z, Y] \text {. }
\end{aligned}
$$

Proof. Given a morphism $f: X \rightarrow Y$ and an approaching fibration $p: E \rightarrow B$ of $f$, consider the diagram $F(f) \rightarrow E \leftarrow X \rightarrow Y$, where $F(f): F(f) \rightarrow \emptyset$ be the "homotopy fibre" of $f$ in $\mathbf{C}_{\emptyset}$. Let $i: F(f) \rightarrow X$ be the morphism in $\operatorname{Ho}(\mathbf{C})$ given by $F(f) \rightarrow E \leftarrow X$ and let $\underline{\hat{i}}: F(f) \rightarrow \emptyset \underline{\times} X$ be the morphism in $\mathrm{Ho}\left(\mathbf{C}_{\emptyset}\right)$ corresponding to $i$ by the adjunction

$$
\operatorname{Ho}\left(\mathbf{C}_{\emptyset}\right) \underset{\emptyset \underline{x}(.)}{\stackrel{V}{\longrightarrow}} \operatorname{Ho}(\mathbf{C})
$$


given in Theorem 3.1 .

Then the diagram $F(f) \rightarrow X \rightarrow Y$ induces the fibration sequence in $\operatorname{Ho}\left(\mathbf{C}_{\emptyset}\right)$

$$
\underline{F(f)} \stackrel{\stackrel{\hat{i}}{\rightarrow}}{\emptyset} \underline{\times} X \stackrel{\emptyset \times f}{\Rightarrow} \emptyset \underline{\times} Y
$$

Since $\mathbf{C}_{\emptyset}$ is a pointed closed model category, we can apply Proposition 2.3 to obtain the exact sequence given in (i).

Using the adjoint functors

$$
\mathrm{Ho}\left(\mathbf{C}_{\emptyset}\right) \stackrel{\Sigma}{\underset{\Omega}{<}} \operatorname{Ho}\left(\mathbf{C}_{\emptyset}\right)
$$

and the previous adjunction one has (ii) .

If $\underline{Z}$ satisfies the hypothesis of (iii), one can apply Corollary 3.1 to obtain the result given in (iii).

Given a morphism $f: X \rightarrow Y$ in $\mathbf{C}$, if we consider a commutative diagram in $\mathbf{C}_{\emptyset}$

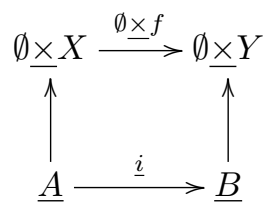

where the vertical maps are trivial fibrations and $\underline{i}$ is a cofibration between cofibrant spaces. We say that $\underline{i}$ is an approaching cofibration of $\emptyset \underline{\times f}$.

Definition 3.2. Given a map $f: X \rightarrow Y$ in $\mathbf{C}$, if $\underline{i}$ is an approaching cofibration of $\emptyset \underline{\times} f$, the cofibre $C(f)$ of $\underline{i}$ given by the push out

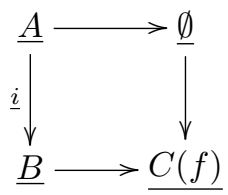

is said the "homotopy cofibre" of $f$ in $\mathbf{C}_{\emptyset}$

If we want to construct a long exact sequence associated to a morphism $f: X \rightarrow$ $Y$ we can proceed as follows:

Theorem 3.4. Let $f: X \rightarrow Y$ be a morphism in a closed model category $\mathbf{C}$. Then for any $Z$ object in $\mathbf{C}$

(i) there is a long exact sequence

$\cdots \rightarrow\left[\Sigma^{q+1}(\emptyset \times X), \emptyset \underline{\times} Z\right]_{\emptyset} \rightarrow\left[\Sigma^{q}(C(f)), \emptyset \underline{\times} Z\right]_{\emptyset} \rightarrow\left[\Sigma^{q}(\emptyset \times Y), \emptyset \times Z\right]_{\emptyset} \rightarrow \cdots$

$\cdots \rightarrow\left[\Sigma(\emptyset \underline{\times X)}, \emptyset \underline{\times} Z]_{\emptyset} \rightarrow[C(f), \emptyset \underline{\times Z}]_{\emptyset} \rightarrow[\emptyset \underline{\times} Y, \emptyset \underline{\times} Z]_{\emptyset} \rightarrow[\emptyset \underline{\times} X, \emptyset \times Z]_{\emptyset}\right.$.

(ii) If $\emptyset$ is a fibrant object $\overline{\text { in } \mathbf{C}}$, then the sequence given at (i) is isomorphic to the exact sequence

$$
\begin{aligned}
& \cdots \rightarrow\left[V \Sigma^{q+1}(\emptyset \times X), Z\right] \rightarrow\left[V \Sigma^{q} C(f), Z\right] \rightarrow\left[V \Sigma^{q}(\emptyset \times Y), Z\right] \rightarrow \cdots \\
& \cdots \rightarrow[V \Sigma(\emptyset \underline{\times} X), Z] \rightarrow[V(\underline{C(f)}), Z] \rightarrow[V(\emptyset \underline{\times} Y), Z] \rightarrow[V(\emptyset \underline{\times} X), Z] .
\end{aligned}
$$


Proof. Since $\emptyset \times \underline{X} \rightarrow \emptyset \underline{\times} Y \rightarrow C(f)$ is a cofibration sequence in $\mathrm{Ho}\left(\mathbf{C}_{\emptyset}\right)$, by the dual of Proposition 2.3 it follows the exact sequence given at (i). To prove (ii), taking into account that $\emptyset$ is fibrant we can apply Theorem 3.1 .

Dually, for a map $f: X \rightarrow Y$ in a closed model category $\mathbf{C}$ we can use the the model structure of $\mathbf{C}^{*}$ to construct more exact sequences associated with $f$.

Given a map $i: A \rightarrow B$ in $\mathbf{C}$, the "cofibre" of $i$ in $\mathbf{C}^{*}$ is the object $\bar{C}: * \rightarrow C$ given by the following pushout

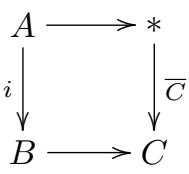

Note that the "cofibre" $\bar{C}$ of $i$ in $\mathbf{C}^{*}$ is isomorphic to the cofibre of $* \bar{\square} i$ in the pointed category $\mathbf{C}^{*}$. On the other hand, if $\bar{f}$ is a morphism in $\mathbf{C}^{*}$, the cofibre of $\bar{f}$ is the "cofibre" of $U \bar{f}$.

For a given map $f: X \rightarrow Y$ in $\mathbf{C}$, we can consider a commutative diagram

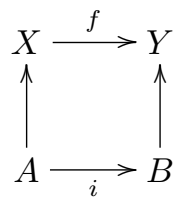

where $i$ is a cofibration, $A, B$ are cofibrant and the vertical maps are trivial fibrations. Then the approaching cofibration $i$ is isomorphic to $f$ in the localized category.

Definition 3.3. Let $\mathbf{C}$ be a closed model category such that $*$ is cofibrant. If $i: A \rightarrow B$ is an approaching cofibration of a morphism $f: X \rightarrow Y$ in $\mathbf{C}$, the "cofibre" of $i$ in $\mathbf{C}^{*}$ is said to be the "homotopy cofibre" of $f$ in $\mathbf{C}^{*}$ and it will be denoted by $\overline{C(f)}$.

As above, all possible "homotopy cofibres" of $f$ in $\mathbf{C}^{*}$ are weak equivalent.

Theorem 3.5. Let $\mathbf{C}$ be a closed model category such that $*$ is cofibrant. Let $f: X \rightarrow Y$ a morphism in $\mathbf{C}$ and let $\overline{C(f)}: * \rightarrow C(f)$ be the "homotopy cofibre" of $f$ in $\mathbf{C}^{*}$ and $\bar{Z}: * \rightarrow Z$ an object in $\mathbf{C}^{*}$. Then

(i) there is a long exact sequence

$\cdots \longrightarrow\left[\Sigma^{q+1}(* \bar{\square} X), \bar{Z}\right]^{*} \rightarrow\left[\Sigma^{q}(\overline{C(f)}), \bar{Z}\right]^{*} \rightarrow\left[\Sigma^{q}(* \bar{\square}), \bar{Z}\right]^{*} \rightarrow \cdots$

$\cdots \rightarrow[\Sigma(* \bar{\square} Y), \bar{Z}]^{*} \rightarrow[\Sigma(* \bar{\square} X), \bar{Z}]^{*} \rightarrow[\overline{C(f)}, \bar{Z}]^{*} \rightarrow[* \bar{\square} Y, \bar{Z}]^{*} \rightarrow[* \bar{\square} X, \bar{Z}]^{*}$

(ii) the sequence given at (i) is isomorphic to the exact sequence

$\cdots \longrightarrow\left[X, U \Omega^{q+1}(\bar{Z})\right] \rightarrow\left[\overline{C(f)}, \Omega^{q} \bar{Z}\right]^{*} \rightarrow\left[Y, U \Omega^{q}(\bar{Z})\right] \rightarrow \cdots$

$\cdots \rightarrow[Y, U \Omega(\bar{Z})] \rightarrow[X, U \Omega(\bar{Z})] \rightarrow[\overline{C(f)}, \bar{Z}]^{*} \rightarrow[Y, U(\bar{Z})] \rightarrow[X, U(\bar{Z})]$

(iii) If $\bar{Z}$ satisfies that $\left[*, U\left(\Omega^{k} \bar{Z}\right)\right]=*$, for $k \geq 0$, then the sequences given at (i) and (ii) are isomorphic to the exact sequence

$\cdots \longrightarrow\left[X, U \Omega^{q+1}(\bar{Z})\right] \rightarrow\left[C(f), U \Omega^{q} \bar{Z}\right] \rightarrow\left[Y, U \Omega^{q}(\bar{Z})\right] \rightarrow \cdots$

$\cdots \rightarrow[Y, U \Omega(\bar{Z})] \rightarrow[X, U \Omega(\bar{Z})] \rightarrow[C(f), U(\bar{Z})] \rightarrow[Y, U(\bar{Z})] \rightarrow[X, U(\bar{Z})]$. 
Given a morphism $f: X \rightarrow Y$ in $\mathbf{C}$, we are going to consider a commutative diagram in $\mathbf{C}^{*}$

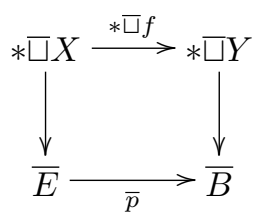

where the vertical maps are trivial cofibrations and $\bar{p}$ is a fibration between fibrant spaces, that is, $\bar{p}$ is an approaching fibration of $* \bar{\square} f$.

Definition 3.4. Given a map $f: X \rightarrow Y$ in $\mathbf{C}$, if $\bar{p}$ is an approaching fibration of $* \square f$, the fibre $\overline{F(f)}$ of $\bar{p}$ given by the pullback

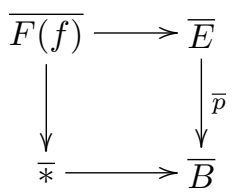

is said the "homotopy fibre" of $f$ in $\mathbf{C}^{*}$

and we have

Theorem 3.6. Let $f: X \rightarrow Y$ be a morphism in a closed model category $\mathbf{C}$. Then for any $Z$ object in $\mathbf{C}$

(i) there is a long exact sequence

$\cdots \rightarrow\left[* \square Z, \Omega^{q+1}(* \square Y)\right]^{*} \rightarrow\left[* \square Z, \Omega^{q}(\overline{F(f)})\right]^{*} \rightarrow\left[* \square Z, \Omega^{q}(* \square X)\right]^{*} \rightarrow \cdots$

$\cdots \rightarrow[* \square Z, \Omega(* \square Y)]^{*} \rightarrow[* \square Z, \overline{F(f)}]^{*} \rightarrow[* \square Z, * \square X]^{*} \rightarrow[* \square Z, * \square Y]^{*}$.

(ii) If $*$ is a cofibrant object in $\mathbf{C}$, then the sequence given at (i) is isomorphic to the exact sequence

$$
\begin{aligned}
& \cdots \rightarrow\left[Z, U \Omega^{q+1}(* \square Y)\right] \rightarrow\left[Z, U \Omega^{q}(\overline{F(f)})\right] \rightarrow\left[Z, U \Omega^{q}(* \square X)\right] \rightarrow \cdots \\
& \cdots \rightarrow[Z, U \Omega(* \square Y)] \rightarrow[Z, U(\overline{F(f)})] \rightarrow[Z, U(* \square X)] \rightarrow[Z, U(* \square Y)] .
\end{aligned}
$$

\section{Applications to Cohomology with Coefficients and group COHOMOLOGY}

Firstly, we include a proof sketch of the following result which gives a homotopy representation of cohomology with local coefficients. We refer the reader to J.M. Møller [27] for a version in terms of mapping spaces.

Theorem 4.1. Let $X$ be a 0-connected $C W$-complex such that $\pi_{1}(X) \cong G$ and consider the canonical map $\underline{X}: X \rightarrow K(G, 1)$. Then for every $n \geq 0$, the $n$-th cohomology group of $X$ with local coefficients in the $G$-module $H$ can be given as the following hom-set in the localized category $\operatorname{Ho}\left(\mathbf{T o p}_{K(G, 1)}\right)$ :

$$
H^{n}(X ; H) \cong \operatorname{Hom}_{\mathrm{Ho}\left(\operatorname{Top}_{K(G, 1)}\right)}(\underline{X}, \underline{K}(G, H ; 1, n))=[\underline{X}, \underline{K}(G, H ; 1, n)]
$$

which is also isomorphic to the corresponding set of fibre (vertical) homotopy classes over $K(G, 1)$.

Proof. For $n \geq 2$, we have the fibration

$$
K(H, n) \stackrel{i_{n}}{\longrightarrow} K(G, H ; 1, n) \stackrel{\rho_{n}}{\longrightarrow} K(G, 1)
$$


and the section $\sigma_{n}: K(G, 1) \rightarrow K(G, H ; 1, n)$. We note that all the spaces are 0 -connected and that for $q \geq 0$ one has the induced bundle of groups

$$
\mathcal{H}_{q}=\left\{\pi_{q}\left(\rho_{n}^{-1}(b), \sigma_{n}(b)\right) \mid b \in K(G, 1)\right\} .
$$

For $q \neq n$ we have a trivial bundle and for $q=n$, one has that $\pi_{n}\left(\rho_{n}^{-1}(b), \sigma_{n}(b)\right) \cong$ $H$. We can suppose that $\pi_{n}\left(\rho_{n}^{-1}\left(b_{0}\right), \sigma_{n}\left(b_{0}\right)\right)=H$, where $b_{0}$ is the base point of $K(G, 1)$. We also note that $\sigma_{n} \underline{X}: X \rightarrow K(G, H ; 1, n)$ is a lifting for the map $\rho_{n}: K(G, H ; 1, n) \rightarrow K(G, 1)$. Now we can apply the Theorem 6.13 of chapter VI of [36] to obtain that

$$
H^{n}(X ; H) \cong[\underline{X}, \underline{K}(G, H ; 1, n)]=\operatorname{Hom}_{\operatorname{Ho}\left(\operatorname{Top}_{K(G, 1)}\right)}(\underline{X}, \underline{K}(G, H ; 1, n))
$$

For $n=1$, one has that $K(G, H ; 1,1)=K(H \rtimes G, 1)$, and we have the fibration

$$
K(H, 1) \stackrel{i_{1}}{\longrightarrow} K(H \rtimes G, 1) \stackrel{\rho_{1}}{\longrightarrow} K(G, 1)
$$

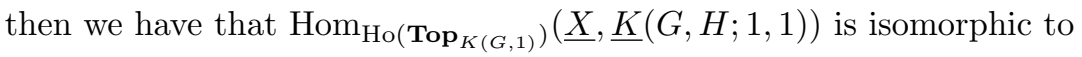

$$
\operatorname{Hom}_{\mathrm{Ho}\left(\left(\mathbf{T o p}^{*}\right)_{K(G, 1)}\right)}\left(\underline{\left(X, x_{0}\right)}, \underline{(K(H \rtimes G, 1), *))}\right) \text { action of } \pi_{1}(K(H, 1), *)
$$

It is easy to check that this is isomorphic to

$$
\operatorname{Der}(G, H) / P(G, H)
$$

where $\operatorname{Der}(G, H)$ is the abelian group of derivations (crossed homomorphism) of $G$ in $H$ and $P(G, H)$ is the group of principal derivations, see section 3 of of chapter VI of [36] or section 2 of chapter IV of [4] . Now we apply Theorem 3.3 of of chapter VI of [36] to obtain that

$$
\operatorname{Der}(G, H) / P(G, H) \cong H^{1}(X ; H)
$$

For $n=0$, one has the covering map

$$
H \stackrel{i_{0}}{\longrightarrow} K(G, H ; 1,0) \stackrel{\rho_{0}}{\longrightarrow} K(G, 1)
$$

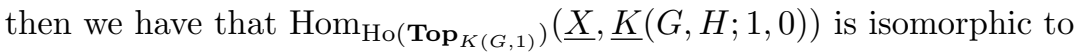

$$
\operatorname{Hom}_{\mathbf{T o p}_{K(G, 1)}}(\underline{X}, \underline{K}(G, H ; 1,0))
$$

Taking into account that $\left.\pi_{1}(K(G, H ; 1,0)), h\right) \cong G_{h}$ and let $H^{G}=\left\{h \in H \mid G_{h}=\right.$ $G\}$. By the usual lifting properties of coverings maps one has that the map $\underline{X}: X \rightarrow K(G, 1)$ has a lifting $\underline{X}^{h}: X \rightarrow K(G, H ; 1,0)$ such that $\underline{X}^{h}\left(x_{0}\right)=h$ if and only if $G_{h}=G$. Therefore $\operatorname{Hom}_{\operatorname{Top}_{K(G, 1)}}(\underline{X}, \underline{K}(G, H ; 1,0))$ is isomorphic to $H^{G}$ and it is well known that $H^{G} \cong H^{0}(X ; H)$.

The final object id: $K(G, 1) \rightarrow K(G, 1)$ in the category $\mathbf{T o p}_{K(G, 1)}$ is denoted by $\underline{K}(G, 1)$. Now, if we take the final object $\underline{K}(G, 1)$ in $\mathbf{T o p}_{K(G, 1)}$, we can consider the category $\operatorname{Top}_{K(G, 1)}^{K(G, 1)}=\operatorname{Top}_{K(G, 1)}^{K(G, 1)}$, the forgetful functor $U: \mathbf{T o p}_{K(G, 1)}^{K(G, 1)} \rightarrow$ $\operatorname{Top}_{K(G, 1)}$ and its left adjoint $\underline{K}(G, 1) \square():. \operatorname{Top}_{K(G, 1)} \rightarrow \operatorname{Top}_{K(G, 1)}^{K(G, 1)}$; see subsection 2.1. The left adjoint carries the object $\underline{X}$ to the object $\underline{K}(G, 1) \square \underline{X}$.

We also have that

$$
K(G, 1) \stackrel{\sigma_{n}}{\longrightarrow} K(G, H ; 1, n) \stackrel{\rho_{n}}{\longrightarrow} K(G, 1)
$$

an object in $\operatorname{Top}_{K(G, 1)}^{K(G, 1)}$, which is denoted by $\underline{\bar{K}}(G, H ; 1, n)$ and the forgetful functor $U: \operatorname{Top}_{K(G, 1)}^{K(G, 1)} \rightarrow \operatorname{Top}_{K(G, 1)}$ verifies that $U(\underline{\bar{K}}(G, H ; 1, n))=\underline{K}(G, H ; 1, n)$. 
With this notation we have the following result:

Theorem 4.2. Let $X$ be a 0-connected $C W$-complex such that $\pi_{1}(X) \cong G$ and consider the canonical map $\underline{X}: X \rightarrow K(G, 1)$. Then for every $n \geq 0$, the $n$-th cohomology group of $X$ with local coefficients in the $G$-module $H$ can be given up to isomorphism as a hom-set in the localized category $\operatorname{Ho}\left(\operatorname{Top}_{K(G, 1)}^{K(G, 1)}\right)$ :

$$
H^{n}(X ; H) \cong \operatorname{Hom}_{\mathrm{Ho}\left(\operatorname{Top}_{K(G, 1)}^{K(G, 1)}\right)}(\underline{K}(G, 1) \square \underline{X}, \underline{\bar{K}}(G, H ; 1, n))
$$

which is also isomorphic to the corresponding set of relative fibre homotopy classes under and over $K(G, 1)$.

Proof. We can apply Theorem 3.2 taking as $\mathbf{C}=\mathbf{T o p}_{K(G, 1)}$ and $A=\underline{K}(G, 1)$ which is a cofibrant object in $\mathbf{T o p}_{K(G, 1)}$ to obtain an adjunction on the localized categories. The result follows from the theorem above and from the existence of this adjunction.

As a particular case of Theorem 4.1 the cohomology of $G$ with coefficients in a $G$-module $H$ can also be interpreted as a set of fibre homotopy classes.

Theorem 4.3. For every $n \geq 0$ the $n$-th cohomology group of $G$ with coefficients in a $G$-module $H$ can be given up to isomorphism as a hom-set in the localized category $\operatorname{Ho}\left(\operatorname{Top}_{K(G, 1)}\right)$

$$
H^{n}(G ; H) \cong[\underline{K}(G, 1), \underline{K}(G, H ; 1, n)]=\operatorname{Hom}_{\mathrm{Ho}\left(\operatorname{Top}_{K(G, 1)}\right)}(\underline{K}(G, 1), \underline{K}(G, H ; 1, n))
$$

which is also isomorphic to the corresponding set of fibre (vertical and non pointed) homotopy classes of sections of the map $\rho_{n}: K(G, H ; 1, n) \rightarrow K(G, 1)$.

Proof. It suffices to take $\underline{X}=\underline{K}(G, 1)$ in Theorem 4.1 and to apply that the cohomology of $K(G, 1)$ with local coefficients in the $G$-module $H$ is isomorphic to the cohomology of $G$ with coefficients in the $G$-module $H$.

Notice that $\underline{K}(G, 1) \square \underline{\underline{K}}(G, 1)$ is isomorphic to $\underline{\bar{K}}(G, 1) \times S^{0}$ where $S^{0}=\{-1,1\}$ and $\sigma: K(G, 1) \rightarrow K(G, 1) \times S^{0}$, is given by $\sigma(b)=(b,-1)$ and $\rho: K(G, 1) \times S^{0} \rightarrow$ $K(G, 1), \rho(b, t)=b$.

Theorem 4.4. For every $n \geq 0$ the $n$-th cohomology group of $G$ with coefficients in a $G$-module $H$ can be given up to isomorphism as a hom-set in the localized category $\operatorname{Ho}\left(\operatorname{Top}_{K(G, 1)}^{K(G, 1)}\right)$ :

$$
H^{n}(G ; H) \cong \operatorname{Hom}_{\mathrm{Ho}\left(\operatorname{Top}_{K(G, 1)}^{K(G, 1)}\right)}\left(\underline{\bar{K}}(G, 1) \times S^{0}, \overline{\bar{K}}(G, H ; 1, n)\right)
$$

which is also isomorphic to the corresponding set of relative fibre homotopy classes under and over $K(G, 1)$.

Proof. Take $\underline{X}=\underline{K}(G, 1)$ in Theorem 4.2 .

Let $\Sigma$ the suspension functor of the closed model category $\operatorname{Ho}\left(\operatorname{Top}_{K(G, 1)}^{K(G, 1)}\right)$, recall that we are thinking on $\Sigma$ as a left derived functor. Since $\underline{\bar{K}}(G, 1) \times S^{0}$ is cofibrant, it easy to check that

$$
\Sigma^{q}\left(\underline{\bar{K}}(G, 1) \times S^{0}\right)=\underline{\bar{K}}(G, 1) \times S^{q}
$$

where the retraction map of $\underline{\bar{K}}(G, 1) \times S^{q}$ is given by the first projection and the section map is induced by the base point of the $q$-sphere. 
Definition 4.1. Let $\underline{\bar{X}}$ be an object in $\operatorname{Top}_{K(G, 1)}^{K(G, 1)}$. For any integer $q \geq 0$, the $q$-th (relative and fibre) homotopy group of $\underline{X}$ is given by

$$
\begin{aligned}
\pi_{q}^{r f}(\underline{\bar{X}}) & =\operatorname{Hom}_{\mathrm{Ho}\left(\operatorname{Top}_{K(G, 1)}^{K(G, 1)}\right)}\left(\Sigma^{q}\left(\underline{\bar{K}}(G, 1) \times S^{0}\right), \underline{\bar{X}}\right) \\
& =\operatorname{Hom}_{\mathrm{Ho}\left(\operatorname{Top}_{K(G, 1)}^{K(G, 1)}\right)}\left(\underline{\bar{K}}(G, 1) \times S^{q}, \underline{\bar{X}}\right)
\end{aligned}
$$

Recall that on the localized category $\operatorname{Ho}(\mathbf{C})$ of a model category $\mathbf{C}$ with zero object $*$ we have a loop functor $\Omega$ that is given as a right derived functor. In the case of a fibrant object $Y$, if $Y^{I}$ denotes a cocylinder of $Y$, the object $\Omega Y$ can given as the fibre of $Y^{I} \times_{Y^{*}} \rightarrow Y$. In the proof of the following proposition the same symbol $\Omega$ is used to denote the loop functors on the localized categories $\operatorname{Ho}\left(\operatorname{Top}_{K(G, 1)}^{K(G, 1)}\right)$ and $\operatorname{Ho}\left(\mathbf{T o p}^{*}\right)$.

Proposition 4.1. For any $G$-module $A$, and $n \geq 0, \Omega(\underline{\bar{K}}(G, A ; 1, n+1))$ is isomorphic in $\operatorname{Ho}\left(\operatorname{Top}_{\mathrm{K}(\mathrm{G}, 1)}^{\mathrm{K}(\mathrm{G}, 1)}\right)$ to $\underline{\bar{K}}(G, A ; 1, n)$.

Proof. The loop functor construction on the model category $\operatorname{Top}_{K(G, 1)}^{K(G, 1)}$ for the fibrant object $\underline{\bar{K}}(G, A ; 1, n+1))$ is given by the fibration sequence

$\Omega(\underline{\bar{K}}(G, A ; 1, n+1)) \rightarrow \underline{\bar{K}}(G, A ; 1, n+1)^{I} \underline{\underline{K}}(G, A ; 1, n+1) \underline{\times} \underline{\bar{K}}(G, 1) \rightarrow \underline{\bar{K}}(G, A ; 1, n)$

Denote

$$
\underline{\overline{P K}}(G, A ; 1, n+1)=\underline{\bar{K}}(G, A ; 1, n+1)^{I} \underline{\bar{K}}(G, A ; 1, n+1) \underline{\times} \underline{\bar{K}}(G, 1)
$$

in this case we can take

$V U \underline{\overline{P K}}(G, A ; 1, n+1)=\left\{u: I \rightarrow K(G, A ; 1, n+1) \mid \rho_{n} u=\right.$ constant, $\left.u(1)=\sigma_{n} \rho_{n}(u(1))\right\}$

and $\rho^{P}: V U \underline{\overline{P K}}(G, A ; 1, n+1) \rightarrow K(G, 1), \sigma^{P}: K(G, 1) \rightarrow V U \underline{\underline{P K}}(G, A ; 1, n+$ 1) are given by $\rho^{P}(u)=\rho_{n}(u(1))$ and $\sigma^{P}(b)(t)=\sigma_{n}(b), b \in \overline{K(G, 1)}, t \in I$. Therefore one has the fibration sequence

$$
\Omega(\underline{\bar{K}}(G, A ; 1, n+1)) \rightarrow \underline{\overline{P K}}(G, A ; 1, n+1) \rightarrow \underline{\bar{K}}(G, A ; 1, n+1)
$$

where $\operatorname{VU} \Omega(\underline{\bar{K}}(G, A ; 1, n+1))$ can be given as

$\left\{u: I \rightarrow K(G, A ; 1, n+1) \mid \rho_{n} u=\right.$ constant, $\left.u(1)=\sigma_{n} \rho_{n}(u(1))=u(0)\right\}$

and $\rho^{\Omega}: \operatorname{VU} \Omega \underline{\bar{K}}(G, A ; 1, n+1) \rightarrow K(G, 1), \sigma^{\Omega}: K(G, 1) \rightarrow V U \Omega \underline{\bar{K}}(G, A ; 1, n+1)$ are given by domain and codomain restrictions of $\rho^{P}$ and $\sigma^{P}$.

Therefore we also have in Top* the fibration

$$
\Omega K(A, n+1) \rightarrow U V \Omega(\underline{\bar{K}}(G, A ; 1, n+1)) \rightarrow K(G, 1)
$$

where the base points are constant paths induced by $b_{0}$ the base point of $K(G, 1)$ and for $n>1, \pi_{n}(\operatorname{VU} \Omega(\underline{\bar{K}}(G, A ; 1, n+1)))$ is isomorphic to $A$ as $G$-modules. For $n=1$, $\pi_{1}(\operatorname{VU} \Omega(\underline{\bar{K}}(G, A ; 1,1+1)))$ is isomorphic to the semidirect product $H \rtimes G$ of the $G$-module $H$ and the group $G$ and for $n=0$, there is a canonical weak equivalence $\Omega(\underline{\bar{K}}(G, A ; 1,1)) \rightarrow \underline{\bar{K}}(G, A ; 1,0)$ in $\operatorname{Top}_{K(G, 1)}^{K(G, 1)}$.

Theorem 4.5. Let $H$ be a $G$-module and $n \geq 0$. Then

(i) $H^{n}(G ; H) \cong \pi_{0}^{r f}(\underline{\bar{K}}(G, H ; 1, n))$, 
(ii) for any integer $q \geq 0, H^{n}(G ; H) \cong \pi_{q}^{r f}(\underline{\bar{K}}(G, H ; 1, n+q))$.

Proof. The first part follows directly from Theorem 4.4 and Definition 4.1 . The second part follows from Proposition 4.1 and the adjunction of the loop and suspension functors.

Remark 4.1. It is interesting to note that in the category under and over $K(G, 1)$ a map induce isomorphism on the Hurewicz homotopy groups if and only if it induces isomorphisms on the cohomology groups with coefficients in any $G$-module $H$. Notice that if we consider the category of $\Omega$-spectra of the form $\{\underline{\bar{K}}(G, H, 1, n)\}$, where $H$ is a $G$-module, the fact that an $\Omega$-spectrum map become an isomorphism can be expressed in terms of group cohomology.

Proposition 4.2. Suppose that $0 \rightarrow H^{\prime} \stackrel{\alpha}{\longrightarrow} H \stackrel{\beta}{\longrightarrow} H^{\prime \prime} \rightarrow 0$ is a short exact sequence of $G$-modules. Then for each $n \geq 0$ the epimorphism $\beta: H \rightarrow H^{\prime \prime}$ induces a fibration map $p_{n}^{\beta}: K(G, H ; 1, n) \rightarrow K\left(G, H^{\prime \prime} ; 1, n\right)$ such that $\rho_{n}^{\prime \prime} p_{n}^{\beta}=\rho_{n}$, $p_{n}^{\beta} \sigma_{n}=\sigma_{n}^{\prime \prime}$ such that if $p_{n}^{\beta} \mid \rho_{n}^{-1}\left(b_{0}\right)$ denotes the restriction to the fibre, one has that for $n \geq 0, \pi_{n}\left(p_{n}^{\beta} \mid \rho_{n}^{-1}\left(b_{0}\right)\right)=\beta$, for $n>1, \pi_{n}\left(p_{n}^{\beta}\right)=\pi_{n}\left(p_{n}^{\beta} \mid \rho_{n}^{-1}\left(b_{0}\right)\right)=\beta$, for $n=1, \pi_{1}\left(p_{1}^{\beta}\right) \cong \beta \rtimes \operatorname{id}_{G}$, and for $n=0, \pi_{0}\left(p_{0}^{\beta}\right) \cong G \backslash \beta$ (the map induced by $\beta$ on the orbit spaces).

If the morphism associated with the map $p_{n}^{\beta}$ is denoted by $\underline{\bar{p}}_{n}^{\beta}: \underline{\bar{K}}(G, H ; 1, n) \rightarrow$ $\underline{\bar{K}}\left(G, H^{\prime \prime} ; 1, n\right)$ in $\operatorname{Top}_{K(G, 1)}^{K(G, 1)}$, we also have that the fibre of the fibration $\underline{p}_{n}^{\beta}$ is isomorphic to $\underline{\bar{K}}\left(G, H^{\prime} ; 1, n\right)$ in $\operatorname{Ho}\left(\operatorname{Top}_{\mathrm{K}(\mathrm{G}, 1)}^{\mathrm{K}(\mathrm{G}, 1)}\right)$.

Proof. For $n=0$ we can apply that the category of pointed covering maps whose fibres are abelian groups over the $\mathrm{CW}$-complex $K(G, 1)$ is equivalent to the category of $G$-modules. Therefore the epimorphism $\beta: H \rightarrow H^{\prime \prime}$ induces a covering transformation $p_{0}^{\beta}: K(G, H ; 1,0) \rightarrow K\left(G, H^{\prime \prime} ; 1,0\right)$. It is easy to check that $p_{0}^{\beta} \sigma_{0}=\sigma_{0}^{\prime \prime}$. Since $\rho_{0}^{\prime \prime} p_{0}^{\beta}=\rho_{0}$ it follows that $p_{0}^{\beta}$ is also a covering map, so $p_{0}^{\beta}$ is a fibration. Using the equivalence functor between the categories, we also have that $\pi_{0}\left(p_{0}^{\beta} \mid \rho_{0}^{-1}\left(b_{0}\right)\right) \cong p_{0}^{\beta} \mid \rho_{0}^{-1}\left(b_{0}\right) \cong \beta$.

For $n=1$, we have that the epimorphism $\beta: H \rightarrow H^{\prime \prime}$ induces the group homomorphisms $\beta \rtimes \operatorname{id}_{G}: H \rtimes G \rightarrow H^{\prime \prime} \rtimes G$. Therefore there is an induced map $f_{1}^{\beta}: K^{\prime}(H \rtimes G, 1) \rightarrow K\left(H^{\prime \prime} \rtimes G, 1\right)$, where $K^{\prime}(H \rtimes G, 1)$ also denotes an Eilenberg-Mac Lane space associated with the semidirect product $H \rtimes G$. Using that $\sigma_{1}$ is a cofibration and $\rho_{1}^{\prime \prime}$ is a fibration in Top we can find a homotopy from $f_{1}^{\beta}$ to $g_{1}^{\beta}$ and the new map satisfies that $g_{1}^{\beta} \sigma_{1}=\sigma_{1}^{\prime \prime}, \rho_{1}^{\prime \prime} g_{1}^{\beta}=\rho_{1}$. In the case $n>1$, since $\beta: H \rightarrow H^{\prime \prime}$ is a $G$-module homomorphism, it is easy to find a map $f_{n}^{\beta}: K^{\prime}(G, H ; 1, n) \rightarrow K(G, H ; 1, n)$, where $K^{\prime}(G, H ; 1, n)$ (also) denotes a CWcomplex with two non trivial homotopy groups $\pi_{1}=G$ and the $G$-module $\pi_{n}=H$. Using that $\sigma_{n}$ is a cofibration and $\rho_{n}^{\prime \prime}$ is a fibration in Top we can find a homotopy from $f_{n}^{\beta}$ to $g_{n}^{\beta}$ and as before the new map satisfies that $g_{n}^{\beta} \sigma_{n}=\sigma_{n}^{\prime \prime}, \rho_{n}^{\prime \prime} g_{1}^{\beta}=\rho_{n}$. Denote by $\underline{\bar{g}}_{n}^{\beta}: \underline{\overline{K^{\prime}}}(G, H ; 1, n) \rightarrow \underline{\bar{K}}\left(G, H^{\prime \prime} ; 1, n\right)$ the corresponding morphism in $\operatorname{Top}_{K(G, 1)}^{K(G, 1)}$.

Next, for $n \geq 1$, we use the factorization properties of the closed model category $\operatorname{Top}_{K(G, 1)}^{K(G, 1)}$ to factor the map $\underline{\bar{g}}_{n}^{\beta}$ as 


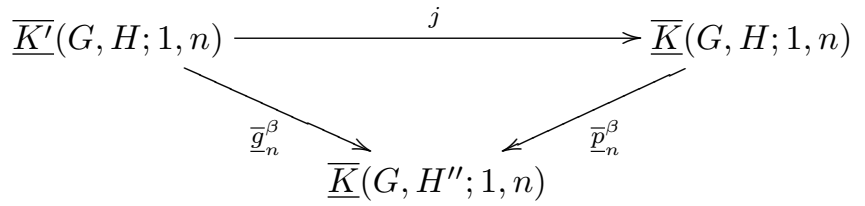

where $j$ is a trivial cofibration and $\underline{\bar{p}}_{n}^{\beta}$ is a fibration.

Therefore for $n \geq 0$, we have the induced fibrations $p_{n}^{\beta}, \underline{p}_{n}^{\beta}$ in Top , Top $\mathbf{T o p}_{K(G, 1)}^{K(G, 1)}$, respectively.

We also note that the space $K(G, 1)$ has a base point $b_{0}$ and then $K(G, 1)$ can also be considered as a pointed space. Therefore one has an induced functor $W: \operatorname{Top}_{K(G, 1)}^{K(G, 1)} \rightarrow \mathbf{T o p}^{*}, W(\underline{\bar{Y}})=\rho_{Y}^{-1}\left(b_{0}\right)$, based at $\sigma_{Y}\left(b_{0}\right)$, where $\rho_{Y}$ is the canonical retraction map and $\sigma_{Y}$ is the section map of the object $\underline{\bar{Y}}$. The functor $W$ preserves fibrations and weak equivalences between fibrant objects. To see that $W$ preserve weak equivalences between fibrant objects you can take a weak equivalence between fibrant objects $\underline{\bar{h}}: \underline{\bar{X}} \rightarrow \underline{\bar{Y}}$ and using the homotopy group sequence of the fibrations $W(\underline{\bar{X}}) \rightarrow X \rightarrow K(G, 1)$ and $W(\underline{\underline{Y}}) \rightarrow Y \rightarrow K(G, 1)$ and the five lemma you obtain that $W(\underline{\underline{h}})$ is a weak equivalence.

The functor $W$ has also a left adjoint functor $K(G, 1) \underline{\underline{ }}():. \mathbf{T o p}^{*} \rightarrow \mathbf{T o p}_{K(G, 1)}^{K(G, 1)}$, which carries the pointed space $X$ to the object $K(G, 1) \underline{\nabla} X$, where the underlying space is the pointed union $K(G, 1) \vee X$ with the obvious retraction and section maps. This functor preserves cofibrations and weak equivalences.

Therefore this pair of adjoint functors induces an adjunction in the localized categories: $(K(G, 1) \underline{\underline{V}}(.))^{L}: \operatorname{Ho}\left(\mathbf{T o p}^{*}\right) \rightarrow \operatorname{Ho}\left(\mathbf{T o p}_{K(G, 1)}^{K(G, 1)}\right)$ and its right adjoint $W^{R}: \operatorname{Ho}\left(\mathbf{T o p}_{K(G, 1)}^{K(G, 1)}\right) \rightarrow \operatorname{Ho}\left(\mathbf{T o p}^{*}\right)$.

Now take the fibre $\underline{F_{n}^{\beta}}$ of $\underline{p}_{n}^{\beta}$ in $\operatorname{Top}_{K(G, 1)}^{K(G, 1)}$ and consider the long sequence

$$
\cdots \rightarrow \Omega\left(\underline{\bar{K}}\left(G, H^{\prime \prime} ; 1, n\right)\right) \rightarrow \underline{\overline{F_{n}^{\beta}}} \rightarrow \underline{\bar{K}}(G, H ; 1, n) \rightarrow \underline{\bar{K}}\left(G, H^{\prime \prime} ; 1, n\right)
$$

Note that

$$
\begin{gathered}
W\left(\underline{\bar{K}}\left(G, H^{\prime \prime} ; 1, n\right)\right) \cong K\left(H^{\prime \prime}, n\right) \\
W(\underline{\bar{K}}(G, H ; 1, n)) \cong K(H, n) \\
W\left(\overline{F_{n}^{\beta}}\right)=\rho_{F_{n}^{\beta}}^{-1}\left(b_{0}\right) \\
W\left(\Omega\left(\underline{\bar{K}}\left(G, H^{\prime \prime} ; 1, n\right)\right)\right) \cong \Omega K\left(H^{\prime \prime}, n\right)
\end{gathered}
$$

and observe that $\operatorname{Hom}_{\mathbf{T o p}_{K(G, 1)}^{K(G, 1)}}\left(K(G, 1) \underline{\nabla} S^{0}, \underline{\bar{X}}\right) \cong W(\underline{\bar{X}})$. Then applying the functor $\operatorname{Hom}_{\mathrm{Ho}\left(\operatorname{Top}_{K(G, 1)}^{K(G, 1)}\right)}\left(\left(K(G, 1) \underline{\nabla}\left(S^{0}\right)\right)^{L},-\right)$ to the sequence above (since $K(G, 1) \underline{\nabla} S^{0}$ is cofibrant, $\left.\left(K(G, 1) \underline{\nabla} S^{0}\right)^{L}=K(G, 1) \underline{\nabla} S^{0}\right)$ and taking into account the adjunction between the suspension and loop functors on $\operatorname{Ho}\left(\operatorname{Top}_{K(G, 1)}^{K(G, 1)}\right)$ and the adjunction above one has the long exact sequence

$$
\cdots \rightarrow \pi_{q+1}\left(K\left(H^{\prime \prime}, n\right)\right) \rightarrow \pi_{q}\left(\rho_{F_{n}^{\beta}}^{-1}\left(b_{0}\right)\right) \rightarrow \pi_{q}(K(H, n)) \rightarrow \pi_{q}\left(K\left(H^{\prime \prime}, n\right)\right) \rightarrow \cdots
$$


and this implies that for $n \geq 0, \rho_{F_{n}^{\beta}}^{-1}\left(b_{0}\right)$ is an Eilenberg Mac Lane space of type $K\left(H^{\prime}, n\right)$ and $\pi_{n}\left(p_{n}^{\beta} \mid \rho_{n}^{-1}\left(b_{0}\right)\right)=\beta$.

Next we consider in Top* the long exact sequence of homotopy groups of the fibration sequence $\rho_{F_{n}^{\beta}}^{-1}\left(b_{0}\right) \rightarrow F_{n}^{\beta} \rightarrow K(G, 1)$ to obtain the long exact sequence

$$
\cdots \rightarrow \pi_{q+1}(K(G, 1)) \rightarrow \pi_{q}\left(\rho_{F_{n}^{\beta}}^{-1}\left(b_{0}\right)\right) \rightarrow \pi_{q}\left(F_{n}^{\beta}\right) \rightarrow \pi_{q}(K(G, 1)) \rightarrow \cdots
$$

For $n>1$ we have that $\pi_{n}\left(F_{n}^{\beta}\right) \cong H^{\prime}$ and $\pi_{1}\left(F_{n}^{\beta}\right) \cong G$ as groups and the other homotopy groups are trivial.

Since the diagram

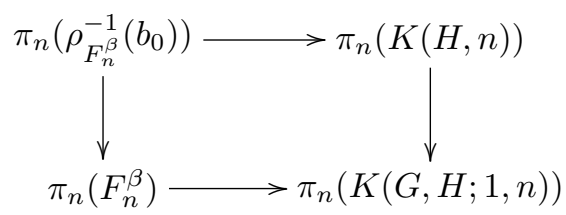

is commutative and $\pi_{n}\left(F_{n}^{\beta}\right) \rightarrow \pi_{n}(K(G, H ; 1, n))$ is a $G$-module homomorphism it follows that $\pi_{n}\left(F_{n}^{\beta}\right) \cong H^{\prime}$ as $G$-modules.

For $n=1$ we obtain the short exact sequence

$$
0 \rightarrow \pi_{1}\left(\rho_{F_{1}^{\beta}}^{-1}\left(b_{0}\right)\right) \rightarrow \pi_{1}\left(F_{1}^{\beta}\right) \rightarrow \pi_{1}(K(G, 1)) \rightarrow 1
$$

Since this sequence splits we can apply Proposition 2.1 of Chapter IV in [4] to obtain that $\pi_{1}\left(F_{1}^{\beta}\right) \cong H^{\prime} \rtimes G$.

For $n=0$ from the construction of the covering maps $K(G, H ; 1,0) \rightarrow K(G, 1)$, $K\left(G, H^{\prime \prime} ; 1,0\right) \rightarrow K(G, 1)$ it follows that $F_{0}^{\beta} \rightarrow K(G, 1)$ is the covering map associated with the $G$-module $H^{\prime}$.

Therefore for $n \geq 0, F_{n}^{\beta}$ is an space of type $K\left(G, H^{\prime} ; 1, n\right)$ and $\overline{F_{n}^{\beta}}$ is isomorphic to $\underline{\bar{K}}\left(G, H^{\prime} ; 1, n\right)$ in $\operatorname{Ho}\left(\mathbf{T o p}_{\mathrm{K}(\mathrm{G}, 1)}^{\mathrm{K}(\mathrm{G}, 1)}\right)$.

Proposition 4.3. Suppose that $0 \rightarrow H^{\prime} \stackrel{\alpha}{\longrightarrow} H \stackrel{\beta}{\longrightarrow} H^{\prime \prime} \rightarrow 0$ is a short exact sequence of $G$-modules. Then there exists a sequence

$\cdots \rightarrow \underline{\bar{K}}\left(G, H^{\prime \prime} ; 1, n-1\right) \rightarrow \underline{\bar{K}}\left(G, H^{\prime} ; 1, n\right) \rightarrow \underline{\bar{K}}(G, H ; 1, n) \rightarrow \underline{\bar{K}}\left(G, H^{\prime \prime} ; 1, n\right) \rightarrow \cdots$ where $n \in \mathbf{Z}($ for $n<0, \quad \underline{\bar{K}}(G, H ; 1, n)$ is the zero object $\underline{\bar{K}}(G, 1))$ and such that for every object $\underline{\bar{X}}$ in $\operatorname{Top}_{K(G, 1)}^{K(G, 1)}$ we have the following exact sequence

$$
\begin{aligned}
& \cdots \rightarrow\left[\underline{\bar{X}}, \underline{\bar{K}}\left(G, H^{\prime \prime} ; 1, n-1\right)\right] \rightarrow\left[\underline{\bar{X}}, \underline{\bar{K}}\left(G, H^{\prime} ; 1, n\right)\right] \quad \rightarrow[\underline{\bar{X}}, \underline{\bar{K}}(G, H ; 1, n)] \rightarrow \\
& \quad \rightarrow\left[\underline{\bar{X}}, \underline{\bar{K}}\left(G, H^{\prime \prime} ; 1, n\right)\right] \rightarrow\left[\underline{\bar{X}}, \underline{\bar{K}}\left(G, H^{\prime} ; 1, n+1\right)\right] \rightarrow \quad \cdots
\end{aligned}
$$

Proof. Given $n \geq 0$ and the epimorphism $\beta: H \rightarrow H^{\prime \prime}$ we can apply Proposition 4.2 to obtain the long sequence

$$
\begin{aligned}
& \cdots \rightarrow \Omega(\bar{K}(G, H ; 1, n)) \rightarrow \Omega\left(\underline{\bar{K}}\left(G, H^{\prime \prime} ; 1, n\right)\right) \rightarrow \\
& \underline{K}\left(G, H^{\prime} ; 1, n\right) \rightarrow \underline{K}(G, H ; 1, n) \rightarrow \underline{K}\left(G, H^{\prime \prime} ; 1, n\right) \rightarrow \cdots
\end{aligned}
$$

Now by Proposition 4.1 , we change $\Omega(\underline{\bar{K}}(G, A ; 1, n))$ by $\underline{\bar{K}}(G, A ; 1, n-1)$ to obtain 


$$
\begin{aligned}
& \cdots \rightarrow \underline{\bar{K}}(G, H ; 1, n-1) \rightarrow \underline{\bar{K}}\left(G, H^{\prime \prime} ; 1, n-1\right) \rightarrow \\
& \underline{\bar{K}}\left(G, H^{\prime} ; 1, n\right) \rightarrow \underline{K}(G, H ; 1, n) \rightarrow \underline{K}\left(G, H^{\prime \prime} ; 1, n\right) \rightarrow \cdots
\end{aligned}
$$

We repeat the argument with $n+1$ and we have the long sequence

$$
\begin{aligned}
& \cdots \rightarrow \underline{\bar{K}}(G, H ; 1, n) \rightarrow \underline{\bar{K}}\left(G, H^{\prime \prime} ; 1, n\right) \rightarrow \\
& \underline{\bar{K}}\left(G, H^{\prime} ; 1, n+1\right) \rightarrow \underline{K}(G, H ; 1, n+1) \rightarrow \underline{K}\left(G, H^{\prime \prime} ; 1, n+1\right) \rightarrow \cdots
\end{aligned}
$$

Since the last written map of the first sequence is the same that the first written map of the second we are able to construct a new sequence changing from one to the next sequence through the common map. In this way, we obtain the sequence given in the thesis of this proposition having the corresponding exactness property.

Theorem 4.6. Let $X$ be a 0-connected $C W$-complex such that $\pi_{1}(X) \cong G$ and consider the canonical map $\underline{X}: X \rightarrow K(G, 1)$. If $0 \rightarrow H^{\prime} \rightarrow H \rightarrow H^{\prime \prime} \rightarrow 0$ is a short exact sequence of $G$-modules, then the long exact sequence associated with the object $\underline{K}(G, 1) \square \underline{X}$ of $\operatorname{Top}_{K(G, 1)}^{K(G, 1)}$ :

$$
\begin{aligned}
& \cdots \rightarrow\left[\underline{K}(G, 1) \square \underline{\square}, \underline{\bar{K}}\left(G, H^{\prime \prime} ; 1, n-1\right)\right] \rightarrow \quad\left[\underline{K}(G, 1) \square \underline{\square}, \underline{\bar{K}}\left(G, H^{\prime} ; 1, n\right)\right] \\
& \rightarrow[\underline{K}(G, 1) \bar{\square}, \underline{X}(G, H ; 1, n)] \rightarrow \quad \rightarrow\left[\underline{K}(G, 1) \bar{\square} \underline{X}, \underline{\bar{K}}\left(G, H^{\prime \prime} ; 1, n\right)\right] \rightarrow \cdots
\end{aligned}
$$

is isomorphic to the long exact sequence

$$
\begin{aligned}
\cdots \rightarrow\left[\underline{X}, \underline{K}\left(G, H^{\prime \prime} ; 1, n-1\right)\right] & \rightarrow\left[\underline{X}, \underline{K}\left(G, H^{\prime} ; 1, n\right)\right] \\
\rightarrow[\underline{X}, \underline{K}(G, H ; 1, n)] & \rightarrow\left[\underline{X}, \underline{K}\left(G, H^{\prime \prime} ; 1, n\right)\right] \rightarrow \cdots
\end{aligned}
$$

which is isomorphic to the long exact sequence of cohomology groups of $X$ with local coefficients in the short exact sequence

$$
\cdots \rightarrow H^{n-1}\left(X ; H^{\prime \prime}\right) \rightarrow H^{n}\left(X ; H^{\prime}\right) \rightarrow H^{n}(X ; H) \rightarrow H^{n}\left(X ; H^{\prime \prime}\right) \rightarrow \cdots
$$

Proof. Firstly take $\underline{K}(G, 1) \square \underline{X}$ in Proposition 4.3 , secondly apply Theorem 3.2 to obtain the second long exact sequence and finally apply Theorem 4.1 to get the last exact sequence.

Remark 4.2. If we take $\underline{\bar{X}}=\underline{K}(G, 1) \times S^{q}$ and change $n$ by $n+q$ in Proposition 4.3, and afterwards apply Theorem 4.5 to obtain the long exact sequence of cohomology groups of a group with coefficients in a short exact sequence $0 \rightarrow H^{\prime} \rightarrow$ $H \rightarrow H^{\prime \prime} \rightarrow 0$ of $G$-modules. Then for any $q \geq 0$ we obtain that the long exact sequence

$$
\begin{aligned}
\cdots \rightarrow \pi_{q}^{r f}\left(\underline{\bar{K}}\left(G, H^{\prime \prime} ; 1, n-1+q\right)\right) & \rightarrow \pi_{q}^{r f}\left(\underline{\bar{K}}\left(G, H^{\prime} ; 1, n+q\right)\right) \\
\rightarrow \pi_{q}^{r f}(\underline{\bar{K}}(G, H ; 1, n+q)) & \rightarrow \pi_{q}^{r f}\left(\underline{\bar{K}}\left(G, H^{\prime \prime} ; 1, n+q\right)\right) \rightarrow \cdots
\end{aligned}
$$

is isomorphic to the long exact sequence of cohomology groups of the group $G$

$$
\cdots \rightarrow H^{n-1}\left(G ; H^{\prime \prime}\right) \rightarrow H^{n}\left(G ; H^{\prime}\right) \rightarrow H^{n}(G ; H) \rightarrow H^{n}\left(G ; H^{\prime \prime}\right) \rightarrow \cdots
$$




\section{Applications to Brown-Grossmann and Steenrod homotopy groups}

By the results of section 2, the categories $\mathbf{E}^{\mathbb{R}_{+}}$and $\mathbf{E}_{\mathbb{R}_{+}}^{\mathbb{R}_{+}}$inherits from $\mathbf{E}$ the induced closed model structures. The corresponding localized categories will be denoted by $\operatorname{Ho}\left(\mathbf{E}^{\mathbb{R}_{+}}\right)$and $\operatorname{Ho}\left(\mathbf{E}_{\mathbb{R}_{+}}^{\mathbb{R}_{+}}\right)$. An object $\underline{\bar{X}}$ in $\mathbf{E}_{\mathbb{R}_{+}}^{\mathbb{R}_{+}}$will usually be denoted by a triple $(X, \sigma, l)$ where $\sigma: \mathbb{R}_{+} \rightarrow X$ and $l: X \rightarrow \mathbb{R}_{+}$are exterior maps such that $l \sigma=\operatorname{id}_{\mathbb{R}_{+}}$, and a morphism in $\mathbf{E}_{\mathbb{R}_{+}}^{\mathbb{R}_{+}}$given by a commutative diagram in $\mathbf{E}$

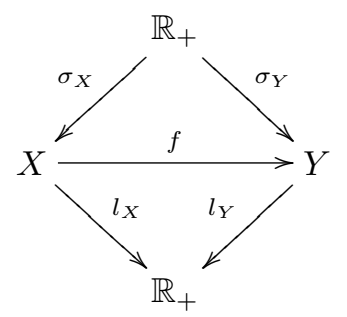

will be denoted by $f:\left(X, \sigma_{X}, l_{X}\right) \rightarrow\left(Y, \sigma_{Y}, l_{Y}\right)$.

Recall that the initial object of $\mathbf{E}$ is the exterior space $\emptyset$ and the final object is $*$ with the trivial externology $\varepsilon_{*}=\{*\}$. In the category $\mathbf{E}^{\mathbb{R}_{+}}$the initial object is $\overline{\mathbb{R}}_{+}=\left(\mathbb{R}_{+}, \operatorname{id}_{\mathbb{R}_{+}}\right)$and the final object is $\bar{*}=\left(*, \sigma_{*}\right)$. The category $\mathbf{E}_{\mathbb{R}_{+}}^{\mathbb{R}_{+}}$is pointed and the zero object is $\overline{\mathbb{R}_{+}}=\left(\mathbb{R}_{+}, \operatorname{id}_{\mathbb{R}_{+}}, \operatorname{id}_{\mathbb{R}_{+}}\right)$. We remark that in $\mathbf{E}$ and in $\mathbf{E}^{\mathbb{R}_{+}}$ all the objects are fibrant.

Let in: $\mathbb{N} \rightarrow \mathbb{R}_{+}$the inclusion. For $q \geq 0$ let $S_{B}^{q}$ be the exterior space obtained by the following pushout in $\mathbf{E}$

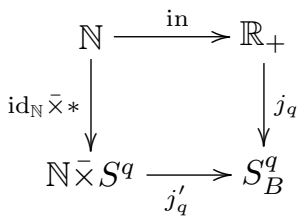

Let $p_{q}: \mathbb{N} \overline{\times} S^{q} \rightarrow \mathbb{R}_{+}$be the map $p_{q}(k, x)=k$ for $(k, x) \in \mathbb{N} \overline{\times} S^{q}$ and $\operatorname{pr}_{q}: S_{B}^{q} \rightarrow$ $\mathbb{R}_{+}$the induced exterior map $\operatorname{pr}_{q}=p_{q}+\operatorname{id}_{\mathbb{R}_{+}}$. Then we denote by $\overline{S_{B}^{q}}=\left(S_{B}^{q}, j_{q}\right)$ and $\overline{S_{B}^{q}}=\left(S_{B}^{q}, j_{q}, \operatorname{pr}_{q}\right)$ the corresponding objects in $\mathbf{E}^{\mathbb{R}_{+}}$and $\mathbf{E}_{\mathbb{R}_{+}}^{\mathbb{R}_{+}}$, respectively.

Note that $\overline{S_{B}^{q}} \sqcup \overline{S_{B}^{q}}=\left(S_{B}^{q} \sqcup_{\mathbb{R}_{+}} S_{B}^{q}, \mathrm{in}_{1} j_{q}\right)$ is given by the following pushout in $\mathbf{E}$

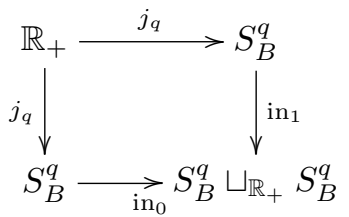

and the cylinder $\overline{S_{B}^{q}} \times I$ of $\overline{S_{B}^{q}}$ in $\mathbf{E}^{\mathbb{R}+}$ is $\left(\left(S_{B}^{q}\right)_{I}, \partial_{1} j_{q}\right)$ where $\left(S_{B}^{q}\right)_{I}$ is the relative cylinder of $S_{B}^{q}$ in $\mathbf{E}$ given by the factorization of $\operatorname{id}_{S_{B}^{q}}+\operatorname{id}_{S_{B}^{q}}$ in $\mathbf{E}$ as the composite of an exterior cofibration and an exterior weak equivalence

$$
S_{B}^{q} \sqcup_{\mathbb{R}_{+}} S_{B}^{q} \stackrel{\partial_{0}+\partial_{1}}{\longrightarrow}\left(S_{B}^{q}\right)_{I} \stackrel{\tau}{\longrightarrow} S_{B}^{q}
$$


Then the suspension $\Sigma\left(\overline{S_{B}^{q}}\right)=\left(L, \mathrm{in}_{1}^{\prime}, \operatorname{pr}_{q} \tau+\mathrm{id}_{\mathbb{R}_{+}}\right)$is the object determined by the pushout in $\mathbf{E}$

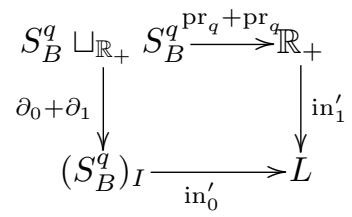

Thus one can see that $\Sigma\left(\underline{\overline{S_{B}^{q}}}\right)$ is isomorphic to $\overline{S_{B}^{q+1}}$ in $\mathbf{E}_{\mathbb{R}_{+}}^{\mathbb{R}_{+}}$.

Analogously, if we denote by $S_{S}^{q}$ the exterior space $\mathbb{R}_{+} \overline{\times} S^{q}$ and by $\overline{S_{S}^{q}}, \overline{S_{S}^{q}}$ the objects in $\mathbf{E}_{\mathbb{R}_{+}}^{\mathbb{R}_{+}}$and $\mathbf{E}^{\mathbb{R}_{+}}$, respectively, given by $\overline{S_{S}^{q}}=\left(S_{S}^{q}, i_{q}, \operatorname{pr}_{q}^{\prime}\right), \overline{S_{S}^{q}}=\left(S_{S}^{q}, i_{q}\right)$, where $i_{q}=\operatorname{id}_{\mathbb{R}_{+}} \overline{\times} *$ and $\operatorname{pr}_{q}^{\prime}(r, x)=r$, for every $(r, x) \in \mathbb{R}_{+} \overline{\times} S^{q}$, we obtain that the suspension $\Sigma\left(\overline{S_{S}^{q}}\right)$ is isomorphic to $\overline{S_{S}^{q+1}}$ in $\mathbf{E}_{\mathbb{R}_{+}}^{\mathbb{R}_{+}}$.

Let $X$ be an exterior space with a base ray $\sigma: \mathbb{R}_{+} \rightarrow X$ and denote $\left.\sigma\right|_{\mathbb{N}}$ the composite $\sigma$ in: $\mathbb{N} \rightarrow X$.

Then, using the closed model category structure of $\mathbf{E}$, the Brown-Grossman and Steenrod exterior homotopy groups can be reinterpreted as the following hom-sets:

$$
\begin{gathered}
\pi_{q}^{B}\left(X,\left.\sigma\right|_{\mathbb{N}}\right) \cong \operatorname{Hom}_{\mathrm{Ho}\left(\mathbf{E}^{\mathbb{R}_{+}}\right)}\left(\left(S_{B}^{q}, j_{q}\right),(X, \sigma)\right), \\
\pi_{q}^{S}(X, \sigma) \cong \operatorname{Hom}_{\mathrm{Ho}\left(\mathbf{E}^{\mathbb{R}_{+}}\right)}\left(\left(S_{S}^{q}, i_{q}\right),(X, \sigma)\right) .
\end{gathered}
$$

Suppose that we have an exterior map $f: X \rightarrow Y$, then we obtain an induced base ray $f \sigma$ in $Y$. Taking in $\mathbf{E}$ the pullback

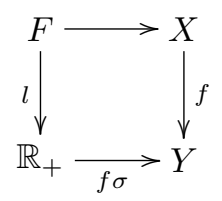

then, the exterior space $F$ is said to be the ray fibre of $f$ with respect to the exterior base ray $\sigma$. Thus we have the object $(F, \omega, l)$ in $\mathbf{E}_{\mathbb{R}_{+}}^{\mathbb{R}_{+}}$, where $\omega=\left(\operatorname{id}_{\mathbb{R}_{+}}, \sigma\right)$.

Applying the results of section 3, we are going to obtain, as in standard homotopy, long exact sequences associated to an exterior fibration.

Theorem 5.1. Let $X$ be an exterior space and $\sigma: \mathbb{R}_{+} \rightarrow X$ a base ray. If $f: X \rightarrow Y$ is an exterior fibration with ray fibre $F$, then there are the following exact sequences:

(i)

$$
\begin{gathered}
\cdots \rightarrow \pi_{q+1}^{B}\left(Y,\left.f \sigma\right|_{\mathbb{N}}\right) \rightarrow \pi_{q}^{B}\left(F,\left.\omega\right|_{\mathbb{N}}\right) \rightarrow \pi_{q}^{B}\left(X,\left.\sigma\right|_{\mathbb{N}}\right) \rightarrow \pi_{q}^{B}\left(Y,\left.f \sigma\right|_{\mathbb{N}}\right) \rightarrow \cdots \\
\quad \cdots \rightarrow \pi_{1}^{B}\left(Y,\left.f \sigma\right|_{\mathbb{N}}\right) \rightarrow \pi_{0}^{B}\left(F,\left.\omega\right|_{\mathbb{N}}\right) \rightarrow \pi_{0}^{B}\left(X,\left.\sigma\right|_{\mathbb{N}}\right) \rightarrow \pi_{0}^{B}\left(Y,\left.f \sigma\right|_{\mathbb{N}}\right)
\end{gathered}
$$

$$
\begin{gathered}
\cdots \rightarrow \pi_{q+1}^{S}(Y, f \sigma) \rightarrow \pi_{q}^{S}(F, \omega) \rightarrow \pi_{q}^{S}(X, \sigma) \rightarrow \pi_{q}^{S}(Y, f \sigma) \rightarrow \cdots \\
\cdots \rightarrow \pi_{1}^{S}(Y, f \sigma) \rightarrow \pi_{0}^{S}(F, \omega) \rightarrow \pi_{0}^{S}(X, \sigma) \rightarrow \pi_{0}^{S}(Y, f \sigma)
\end{gathered}
$$


Proof. Consider the closed model category $\mathbf{C}=\mathbf{E}^{\mathbb{R}_{+}}$whose initial object $\emptyset$ is $\left(\mathbb{R}_{+}, i_{\mathbb{R}_{+}}\right)$. Recall that all objects in $\mathbf{E}^{\mathbb{R}_{+}}$are fibrant. Notice that $f:(X, \sigma) \rightarrow$ $(Y, f \sigma)$ is a morphism in $\mathbf{C}$ whose "homotopy fibre" in $\mathbf{C}_{\emptyset}=\mathbf{E}_{\mathbb{R}_{+}}^{\mathbb{R}_{+}}$is exactly $(F, \omega, l)$.

Taking the object $\underline{\overline{S_{B}^{0}}}$ in $\mathbf{E}_{\mathbb{R}_{+}}^{\mathbb{R}_{+}}$, one has that $V \Sigma^{q}\left(\underline{\overline{S_{B}^{0}}}\right)=\left(S_{B}^{q}, j_{q}\right)$ and

$$
\operatorname{Hom}_{\mathrm{Ho}\left(\mathbf{E}^{\mathbb{R}_{+}}\right)}\left(\left(S_{B}^{q}, j_{q}\right),\left(\mathbb{R}_{+}, \operatorname{id}_{\mathbb{R}_{+}}\right)\right) \cong \pi_{q}^{B}\left(\mathbb{R}_{+},\left.\operatorname{id}_{\mathbb{R}_{+}}\right|_{\mathbb{N}}\right) \cong *
$$

for all $q \geq 0$. Then we can apply (iii) of Theorem 3.3 to obtain the exact sequence

$$
\begin{gathered}
\cdots \rightarrow \operatorname{Hom}_{\mathrm{Ho}\left(\mathbf{E}^{\mathbb{R}_{+}}\right)}\left(\left(S_{B}^{q+1}, j_{q+1}\right),(Y, f \sigma)\right) \rightarrow \operatorname{Hom}_{\mathrm{Ho}\left(\mathbf{E}^{\mathbb{R}_{+}}\right)}\left(\left(S_{B}^{q}, j_{q}\right),(F, \omega)\right) \rightarrow \\
\operatorname{Hom}_{\mathrm{Ho}\left(\mathbf{E}^{\mathbb{R}_{+}}\right)}\left(\left(S_{B}^{q}, j_{q}\right),(X, \sigma)\right) \rightarrow \operatorname{Hom}_{\mathrm{Ho}\left(\mathbf{E}^{\mathbb{R}_{+}}\right)}\left(\left(S_{B}^{q}, j_{q}\right),(Y, f \sigma)\right) \rightarrow \cdots
\end{gathered}
$$

which is isomorphic to the one given in (i) above.

In an analogous way, taking now the object $\overline{S_{S}^{0}}$ in $\mathbf{E}_{\mathbb{R}_{+}}^{\mathbb{R}_{+}}$, one has that $V \Sigma^{q}\left(\overline{S_{S}^{0}}\right)=$ $\left(S_{S}^{q}, i_{q}\right)$ and

$$
\operatorname{Hom}_{\mathrm{Ho}\left(\mathbf{E}^{\mathbb{R}_{+}}\right)}\left(\left(S_{S}^{q}, i_{q}\right),\left(\mathbb{R}_{+}, \operatorname{id}_{\mathbb{R}_{+}}\right)\right) \cong \pi_{q}^{S}\left(\mathbb{R}_{+}, \mathrm{id}_{\mathbb{R}_{+}}\right) \cong *
$$

for all $q \geq 0$. Then we can apply again (iii) of Theorem 3.3 to obtain the exact sequence given in (ii) above.

Next as a consequence of the results of section 3 we are going to prove that the Brown-Grossman and the Steenrod exterior homotopy groups are related by a long exact sequence which is an analogue for exterior spaces of the exact sequence given by Quigley [34] in shape theory or by Porter [29] in proper homotopy theory.

Theorem 5.2. Let $X$ be an exterior space and let $\sigma: \mathbb{R}_{+} \rightarrow X$ be a base ray, then there is an exact sequence

$$
\begin{gathered}
\cdots \rightarrow \pi_{q+1}^{B}\left(X,\left.\sigma\right|_{\mathbb{N}}\right) \rightarrow \pi_{q}^{S}(X, \sigma) \rightarrow \pi_{q}^{B}\left(X,\left.\sigma\right|_{\mathbb{N}}\right) \longrightarrow \pi_{q}^{B}\left(X,\left.\sigma\right|_{\mathbb{N}}\right) \rightarrow \\
\quad \cdots \rightarrow \pi_{1}^{B}\left(X,\left.\sigma\right|_{\mathbb{N}}\right) \rightarrow \pi_{0}^{S}(X, \sigma) \rightarrow \pi_{0}^{B}\left(X,\left.\sigma\right|_{\mathbb{N}}\right) \longrightarrow \pi_{0}^{B}\left(X,\left.\sigma\right|_{\mathbb{N}}\right) .
\end{gathered}
$$

Proof. We consider the exterior map sh: $S_{B}^{0} \rightarrow S_{B}^{0}$ given by $\operatorname{sh}\left(j_{0}(t)\right)=j_{0}(t)$ for every $t \in \mathbb{R}_{+}$and $\operatorname{sh}\left(j_{0}^{\prime}(n, 1)\right)=j_{0}^{\prime}(n+1,1), \operatorname{sh}\left(j_{0}^{\prime}(n,-1)\right)=j_{0}^{\prime}(n,-1)$ for every $n \in \mathbb{N}$.

Let

$$
\overline{\mathrm{sh}}: \overline{S_{B}^{0}}=\left(S_{B}^{0}, j_{0}\right) \rightarrow \overline{S_{B}^{0}}=\left(S_{B}^{0}, j_{0}\right)
$$

be the corresponding morphism in the $\mathbf{E}^{\mathbb{R}_{+}}$. Next we study its "homotopy cofibre" $\underline{C(\overline{\mathrm{sh}})}$ in $\mathbf{E}_{\mathbb{R}_{+}}^{\mathbb{R}_{+}}$defined in Definition 3.2. Note that the functor $\overline{\mathbb{R}_{+}} \underline{x}():. \mathbf{E}^{\mathbb{R}_{+}} \rightarrow$

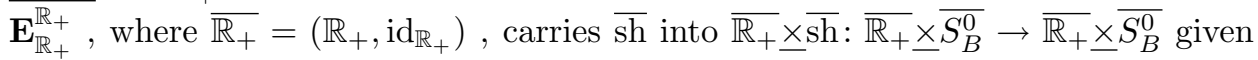
by the commutative diagram

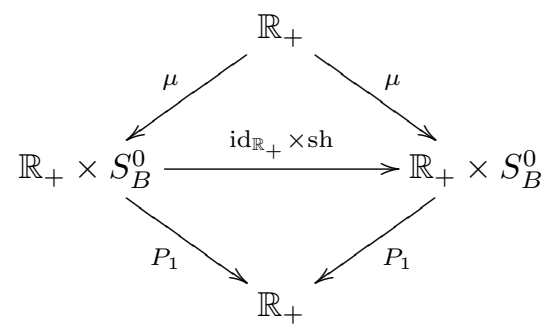


where $\mu=\left(\operatorname{id}_{\mathbb{R}_{+}}, j_{0}\right)$ and $P_{1}$ is the projection $P_{1}(t, x)=t$ for every $(t, x) \in \mathbb{R}_{+} \times S_{B}^{0}$. We remark that $\overline{\mathbb{R}_{+}} \times \overline{s h}$ is a cofibration between cofibrant objects in $\mathbf{E}_{\mathbb{R}_{+}}^{\mathbb{R}_{+}}$, so $\underline{C(\overline{\operatorname{sh}})}$ is the object given by the triple $\left(C, i n_{1}, P_{1}+\mathrm{id}_{\mathbb{R}_{+}}\right)$, where $C$ is given by the pushout diagram in $\mathbf{E}$

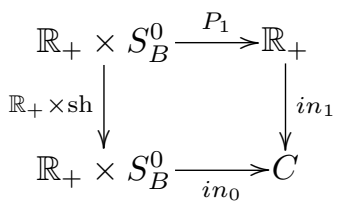

Therefore $\underline{C(\overline{\mathrm{sh}})}$ is isomorphic in $\mathbf{E}_{\mathbb{R}_{+}}^{\mathbb{R}_{+}}$to $\overline{S_{S}^{0}}=\left(S_{S}^{0}, i_{0}, \mathrm{pr}_{0}^{\prime}\right)$.

On the other hand, the morphism in $\mathbf{E}_{\mathbb{R}_{+}}^{\mathbb{R}_{+}}$

$$
d: \overline{\overline{S_{B}^{0}}}=\left(S_{B}^{0}, j_{0}, \mathrm{pr}_{0}\right) \rightarrow \overline{\mathbb{R}_{+}} \times \overline{S_{B}^{0}}=\left(\mathbb{R}_{+}, \mu, P_{1}\right)
$$

given by $d\left(j_{0}(t)\right)=\mu(t)$ for every $t \in \mathbb{R}_{+}, d\left(j_{0}^{\prime}(n, x)\right)=\left(n, j_{0}^{\prime}(n, x)\right)$ for every $(n, x) \in \mathbb{N} \times S^{0}$, is a trivial cofibration between cofibrant objects in $\mathbf{E}_{\mathbb{R}_{+}}^{\mathbb{R}_{+}}$. Therefore for $q \geq 0$ the suspension $\Sigma^{q}\left(\overline{\mathbb{R}_{+}} \times \overline{S_{B}^{0}}\right)$ is isomorphic to $\overline{S_{B}^{q}}$ in $\operatorname{Ho}\left(\mathbf{E}_{\mathbb{R}_{+}}^{\mathbb{R}_{+}}\right)$, so $V\left(\Sigma^{q}\left(\overline{\mathbb{R}_{+}} \times \overline{S_{B}^{0}}\right)\right)$ is isomorphic to $\overline{S_{B}^{q}}$ in $\operatorname{Ho}\left(\mathbf{E}^{\mathbb{R}_{+}}\right)$.

Now suppose that $X$ is an exterior space and $\sigma: \mathbb{R}_{+} \rightarrow X$ a base ray in $X$. We can apply (ii) of Theorem 3.4 to the category $\mathbf{C}=\mathbf{E}^{\mathbb{R}_{+}}$and to the morphism $\overline{\mathrm{sh}}: \overline{S_{B}^{0}} \rightarrow \overline{S_{B}^{0}}$ and taking into account the remarks above we obtain the long exact sequence

$$
\begin{array}{r}
\left.\left.\cdots \rightarrow \operatorname{Hom}_{\mathrm{Ho}\left(\mathbf{E}^{\mathbb{R}}+\right)}\left(\left(S_{B}^{q+1}, j_{q+1}\right)\right),(X, \sigma)\right) \longrightarrow \operatorname{Hom}_{\mathrm{Ho}\left(\mathbf{E}^{\mathbb{R}}+\right)}\left(\left(S_{S}^{q}, i_{q}\right)\right),(X, \sigma)\right) \longrightarrow \\
\left.\left.\operatorname{Hom}_{\mathrm{Ho}\left(\mathbf{E}^{\mathbb{R}^{+}}\right)}\left(\left(S_{B}^{q}, j_{q}\right)\right),(X, \sigma)\right) \stackrel{\overline{\mathrm{sh}}_{*}}{\longrightarrow} \operatorname{Hom}_{\mathrm{Ho}\left(\mathbf{E}^{\mathbb{R}}+\right)}\left(\left(S_{B}^{q}, j_{q}\right)\right),(X, \sigma)\right) \longrightarrow \\
\left.\cdots \operatorname{Hom}_{\mathrm{Ho}\left(\mathbf{E}^{\mathbb{R}}+\right)}\left(\left(S_{B}^{1}, j_{1}\right)\right),(X, \sigma)\right) \longrightarrow \\
\left.\left.\operatorname{Hom}_{\mathrm{Ho}\left(\mathbf{E}^{\mathbb{R}}+\right)}\left(\left(S_{B}^{0}, j_{0}\right)\right),(X, \sigma)\right) \stackrel{\overline{\mathrm{sh}}_{*}}{\longrightarrow} \operatorname{Hom}_{\mathrm{Ho}\left(\mathbf{E}^{\mathbb{R}}+\right)}\left(\left(S_{S}^{0}, i_{0}\right)\right),(X, \sigma)\right) \longrightarrow \\
\end{array}
$$

which is isomorphic to the long exact sequence given in the thesis of the theorem.

\section{REFERENCES}

[1] V. Alvarez, J.A. Armario and P. Real, On the computability of the p-local homology of twisted cartesian products of Eilenberg-Mac Lane spaces, in: First Meeting on Geometry and Topology, Braga (1997).

[2] H.J. Baues. Algebraic homotopy. Cambridge Univ. Press, 1988.

[3] H.J. Baues and A. Quintero. Infinite Homotopy Theory, K-monographs in Mathematics 6, June 2001, Hardbound.

[4] K.S. Brown. Cohomology of groups, Springer-Verlag, GTM 87, 1982.

[5] E.M. Brown. On the proper homotopy type of simplicial complexes, Topology Conference, Lect. Notes in Math., no. 375, Springer-Verlag, New York, 1975, pp. 41-46. 
[6] E.M. Brown and T.W. Tucker, On proper homotopy theory for non compact 3-manifolds, Trans. Amer. Math. Soc. 188 (1977), no. 7, 105-126.

[7] C. Casacuberta, L.J. Hernández and J.L. Rodríguez. Models for torsion homotopy types, Israel J. of Math. 107 (1998), 301-318.

[8] J.M. Cordier and T. Porter. Shape theory, categorical methods of approximation, Ellis Horwood Series in Math and its Applications, 1989.

[9] W. G. Dwyer and J. Spalinski. Homotopy theories and model categories, Handbook of Algebraic Topology, pp. 73-126, Elsevier Science B. V., Amsterdam, 1995.

[10] W.G. Dwyer, P. S. Hirschhorn, D.M. Kan and J. Smith Homotopy Limit Functors on Model Categories and Homotopical Categories, Mathematical Surveys and Monographs, AMS, vol. 113, 2005.

[11] D. Edwards and H. Hastings. Čech and Steenrod homotopy theories with applications to Geometric Topology, Lect. Notes Math., 542 (Springer, 1976).

[12] J. I. Extremiana, L. J. Hernández, and M. T. Rivas, An isomorphism theorem of the Hurewicz type in the proper homotopy category, Fund. Math. 132 (1989), 195-214.

[13] J. I. Extremiana, L. J. Hernández and M. T. Rivas. A closed model category for $(n-1)$ connected spaces, Proc. Amer. Math. Soc. 124 (1996), 3545-3553.

[14] H. Freudenthal. Über die Enden topologisher Räume und Gruppen, Math. Zeith., 53 (1931), 692-713.

[15] M. H. Freedman. The topology of four-dimensional manifolds, J. Diff. Geom., 17 (1982), 357-453.

[16] P. Gabriel and M. Zisman. Calculus of fractions and homotopy theory. Springer, Berlin (1966).

[17] J.M. García, M. García and L.J. Hernández. A closed simplicial model category for homotopy and shape theories, Bull. Austral. Math. Soc. 57 (1998), 221-242.

[18] J.M. García, M. García and L.J. Hernández. Closed simplicial model structures for exterior and proper homotopy theory, Appl. Cat. Struct., 12 (3), ( 2004) 225-243.

[19] J.M. García-Calcines, P. R. García-Díaz and A. Murillo-Mas. A Whitehead-Ganea approach for proper Lusternik-Schnirelmann category, Math. Proc. Camb. Phil. Soc. 142/3 (2007), 439-457.

[20] P. G. Goerss and J. F. Jardine. Simplicial homotopy theory, Progress in mathematics, no. 174, Birkhäuser Verlag, Basel-Boston-Berlin, 1999.

[21] J.W. Grossman. Homotopy groups of pro-spaces, Illinois J. Math. 20 (1976), 622-625.

[22] L.J. Hernández. Application of simplicial M-sets to proper homotopy and strong shape theories, Transactions of the A.M.S. 347 (1995), no. 2, 363-409.

[23] L.J. Hernández. Closed model categories for uniquely S-divisible spaces, J. Pure and Appl. Alg 182 (2003), 223-237.

[24] P.S. Hirschhorn. Model categories and their localizations, Mathematical Surveys and Monographs, vol. 99, Am. Math. Soc., 2003.

[25] M. Hovey. Model categories, Mathematical Surveys and Monographs 63, AMS, Providence, RI, 1999 ( $\mathrm{x}+209$ pages).

[26] S. Mardešić and J. Segal. Shape theory. (North-Holland, 1982).

[27] J. M. Møller. Spaces of sections of Eilenberg-Mac Lane fibrations, Pacific J. Math. 130 (1987), pp. 171-186.

[28] T. Porter. Proper homotopy theory, Handbook of Algebraic Topology (ch. 3) (1995), 127-167.

[29] T. Porter. Cech and Steenrod homotopy and the Quigley exact couple in strong shape and proper homotopy theory, J. Pure and Appl. Alg. 24 (1983), 303-312.

[30] L.C. Siebenmann, The obstruction to finding a boundary for an open manifold of dimension greater than five, PhD thesis, 1965.

[31] L.C. Siebenmann, Infinite simple homotopy types, Indag. Math., 32 (1970), 479-495.

[32] D. Quillen. Homotopical Algebra, Lect. Notes in Math, 43 (Springer, 1967).

[33] D. Quillen. Rational Homotopy Theory, Ann. of Math. 90 (1969), 205-295.

[34] J.B. Quigley. An exact sequence from the $n$-th to the (n-1)-st fundamental group, Fund. Math. 77 (1973), 195-210.

[35] K. Tsuchida. Generalized Puppe sequence and Spanier-Whitehead duality. Tôcutu Math. Journ 23 (1971), pp. 37-48.

[36] G. W. Whitehead. Elements of Homotopy Theory, Springer-Verlag, 1995. 
Mónica García Pinillos, Departamento de Matemáticas. Universidad de la Rioja, 26004, Logroño, SPAIN.

E-mail address: marque11@centros4.pntic.mec.es

Luis Javier Hernández Paricio, Departamento de Matemáticas. Universidad de La Rioja, 26004, Logroño, Spain.

E-mail address: luis-javier.hernandez@unirioja.es

María Teresa Rivas Rodríguez, Departamento de Matemáticas. Universidad de La Rioja, 26004, Logroño, Spain.

E-mail address: maria-teresa.rivas@unirioja.es 